\title{
X-ray powder diffraction in reflection geometry on multi-beam kJ-type laser facilities
}

\author{
A. Denoeud ${ }^{1, \mathrm{a})}$, J.-A. Hernandez ${ }^{2,3}$, T. Vinci ${ }^{2}$, A. Benuzzi-Mounaix ${ }^{2}$, S. Brygoo ${ }^{1}$, A. Berlioux ${ }^{2}$, F. Lefevre ${ }^{2}$, A. \\ Sollier $^{1,4}$, L. Videau ${ }^{1,4}$, A. Ravasio ${ }^{2}$, M. Guarguaglini ${ }^{2}$, L. Duthoit ${ }^{1}$, D. Loison ${ }^{5}$, E. Brambrink ${ }^{2,6}$ \\ ${ }^{1}$ CEA, DAM, DIF, F-91297 Arpajon, France \\ ${ }^{2}$ LULI, CNRS, CEA, Sorbonne Université, École Polytechnique, Institut Polytechnique de Paris, F-91128 \\ Palaiseau, France \\ ${ }^{3}$ Centre for Earth Evolution and Dynamics, University of Oslo, Box 1028 Blindern, N-0315 Oslo, Norway \\ ${ }^{4}$ Université Paris-Saclay, CEA, Laboratoire Matière en Conditions Extrêmes, 91680 Bruyères-le-Châtel, France \\ ${ }^{5}$ Univ Rennes, CNRS, IPR (Institut de Physique de Rennes) - UMR 6251, F-35000 Rennes, France \\ ${ }^{6}$ European XFEL, Holzkoppel 4, 22869, Schenefeld, Germany
}

a)Author to whom correspondence should be addressed: adrien.denoeud2@cea.fr

\begin{abstract}
An ultrafast X-ray powder diffraction setup for laser-driven dynamic compression has been developed at LULI2000 laser facility. X-ray diffraction is performed in reflection geometry from a quasi-monochromatic lasergenerated plasma X-ray source. In comparison to a transmission geometry setup, this configuration allows to probe only a small portion of the compressed sample, as well as to shield the detectors against the X-rays generated by the laser-plasma interaction on the front side of the target. Thus, this new platform facilitates probing of spatially and temporarily uniform thermodynamic conditions and enables to study samples on a large range of atomic numbers, thicknesses and compression dynamics. As a proof-of-concept, we report direct structural measurements of the bcc-hep transition both in shock and ramp-compressed polycrystalline iron with diffraction signals recorded between $2 \theta \sim 30^{\circ}$ and $\sim 150^{\circ}$. In parallel, the pressure and temperature history of probed samples is measured by rear-side visible diagnostics (velocimetry, pyrometry).
\end{abstract}

\section{Introduction}

Dynamic compression experiments are increasingly used to determine the properties of solids and liquids from few GPa and hundreds of Kelvin up to several TPa and several thousands of Kelvin $[1,2]$. However, the short time-scales, i.e. from hundred picoseconds to few microseconds, have precluded direct in situ structural measurements for a long-time. That is especially true for diagnostics that required the use of X-ray beams, as Xray radiography, X-ray absorption Fine Structure (XAFS) or X-ray diffraction (XRD). The latter is particularly interesting, since it enables us to study the ionic structural changes in solids without requiring any link through complex modules such as ab initio calculations [3-5]. With fast X-ray diffraction, one can thus have access directly to the dynamical phase diagram of materials, identify their melting curves, or even study the dynamic evolution of their different phase transitions.

During the last two decades, the development of intense pulsed ultrafast X-ray sources at synchrotron facilities or the rise of X-ray free electron lasers (XFEL), coupled with small gas-guns or multi-joule nanosecond lasers, have enabled direct probing of the structure of dynamically compressed matter up to a few Mbar [6-15]. Nevertheless, while these facilities benefit from very intense, ultrashort, monochromatic X-ray sources, they do not have the drive capabilities of large gas-guns, Z-machines, or (multi-)kilojoules laser facilities yet, in terms of 
energy and pulse shaping. Indeed, this type of facilities are the only tool today to get important data, including equation of state and structural information, in the range of multi-Mbar and temperatures $\approx 0.1-1 \mathrm{eV}$, of interest for Warm Dense Matter and planetology studies [16-18], as well as for phase transition dynamics studies under high pressure ramp compression [19-21].

For this reason, laser-based plasma X-ray sources have been developed in large laser facilities. The interaction of a (sub-)nanosecond laser pulse at intensities on target of typically $10^{14}-10^{17} \mathrm{~W} / \mathrm{cm}^{2}$ with a solid target, usually referred as backlighter, produces a plasma emitting quasi-monochromatic hard X-ray radiations $\left(\mathrm{He}_{\alpha}, \mathrm{K}_{\alpha}\right)$ due to electronic transitions [22-25]. Synchronization and collimation of this bright spherical emission with dynamic compression allows performing in-situ ultrafast X-ray diffraction of stressed samples. These samples can be compressed via either shock or ramp loading, and its hydrodynamic conditions (pressure and temperature) measured by velocity interferometer system for any reflector (VISAR) [26] and streaked optical pyrometer (SOP) diagnostics [27]. X-rays passing through the sample are then diffracted if they satisfy Bragg's Law: $\lambda=2 \operatorname{dsin} \theta$, where $\lambda$ is the $X$-ray wavelength, $d$ is the interplanar lattice spacing, and $\theta$ is the angle between the incident beam and the lattice plane. Diffracted X-rays are finally recorded on X-ray detectors such as imaging plates, which are, in addition to being practical to use, adaptable in size, relatively inexpensive, usable over a wide energy range, and resistant to radiation, electromagnetic pulses and debris [28-30].

The development of such X-ray diffraction techniques under in-situ dynamic loading on laser facilities began just over twenty years ago [31,32]. The first schemes were simply composed of image plates surrounding the compressed target, as illustrated in Figure 1.a. These experiments succeeded to collect diffraction signal from highly diffracting single crystal then polycrystalline shock-compressed materials up to few tens of GPa [33-35]. Nevertheless, at higher pressure and temperature, the rapid increase of X-ray noise from the drive plasma makes detection of diffracted photons from the sample more challenging. Indeed, the incoherent scattering from the laserablated plasma cannot be neglected anymore, and disturbs severely the collected signal. Moreover, in transmission schemes, because of both the higher velocity of the shock and the quicker release of the shock-compressed samples, the time duration of the X-ray probe must be short enough to avoid integrating inhomogeneous hydrodynamic conditions, which is not the case with ns time scale sources. That is why other diffraction schemes were designed these last years to overcome these issues, and to obtain data at higher pressures in homogeneous hydrodynamic conditions.

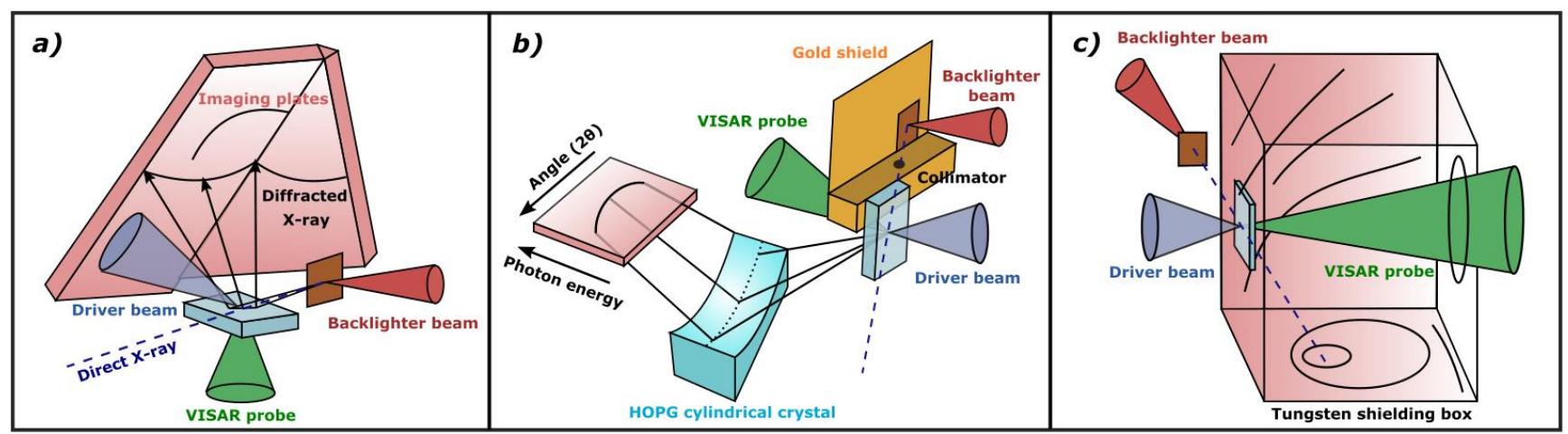

Figure 1: Different experimental setups for laser-driven dynamic-compression experiments with X-ray diffraction. The main target, which contains the sample, and the backlighter target are represented in sky blue and in brown respectively. Driver, backlighter and VISAR probe beams are represented in blue, red and green respectively. Diffracted black lines are printed on pink imaging plates. In the scheme a), adapted from [33], X-rays scattered by the sample are directly recorded on image plates that surrounded it. This scheme is reproduced with permission from Phys. Rev. Letters, 95 (2005). Copyright 2005, American Physical Society. In the scheme b), adapted from [36], X-rays, which are diffracted by the rear side of the shocked sample, are selected in energy thanks to an 
analyzer crystal. In the scheme c), adapted from [39] with the permission of AIP Publishing, diffracted X-rays passing through the main target are recorded in a shielded box. In each scheme, a VISAR laser probe is focused onto the rear surface of the target package to measure or deduce the probed hydrodynamical conditions.

The coupling between a large analyzer crystal and a gold shielding, as illustrated in Figure 1.b, allowed increasing the signal-to-noise ratio by separating coherent from incoherent scattering and direct radiation from the drive-laser sample interaction [36]. The use of such an angle-resolved Von Hamos spectrometer could significantly reduce the uncertainties in identifying the elastic signal contribution, and thus increase accuracy in structure determination of materials shock-compressed over hundred GPa. Moreover, in this reflection geometry, adjustment of the sample thickness and of the pump-probe delay allowed to control accurately the pressure and temperature conditions, despite the low temporal resolution of few ns. Indeed, the diffracted X-rays collected by the crystal came mostly from the first microns of the target's rear side, which are unshocked before being homogeneously compressed. Nevertheless, this technique can only collect diffracted signal over a limited range of $(2 \theta, \phi)$ angles. This considerably limits the nature of the studied materials, which can only be polycrystalline. In the same spirit, a diffraction device spectrally resolved by utilizing single photon counting techniques associated with a white X-ray source has also been proposed [37] and tested at low pressure [38] in order to separate coherent from incoherent scattering. However, such a device has similar limitations to the previous setup since it does not have resolution according to the azimuthal angle $\phi$.

Nowadays, a fully-shielded version of the first diffractometer is now routinely used at Omega and NIF laser facilities, as shown in Figure 1.c [39, 40]. This diagnostic, which works in transmission geometry, i.e. with the backlighter placed on the drive laser side of the target, has made it possible to extend studies to TPa pressures [41-47]. In this configuration, without any X-ray collimator than the pinhole placed directly on the main target, the diffracted signal includes structural information of the entire target depth. This implies that thermodynamic conditions have to be homogeneous in the whole volume of the sample during X-ray probing. That is why this platform is essentially used to study ramp-compressed thin sample, surrounded by diamonds anvil, whose compression state could be steadied during few ns. However, this may be an issue for time resolved studies, or for associating a given structure inferred from XRD to a particular time of the compression history. Moreover, hard $\mathrm{X}$-rays produced by the ablation coronal plasmas of both targets cannot be completely blocked by the main target, which must at least be transparent to the probing X-ray. This means that diffracted measurements are inevitably disturbed by a diffused hard X-ray noise, not to mention that the extremely bright 0 -order is directly collected by the image plates. In such large multi-beam facilities as Omega and NIF, these problems are overridden by the use of very bright laser-plasma X-ray source, or by limiting the peak intensity and thus peak pressure to reduce X-ray emission in the ablation plasma. However, it is not solved for the study of weak diffracting or strong X-ray absorbing materials, or to extend these studies to smaller laser facilities.

In order to overcome these various limitations, we coupled the two last techniques described above, i.e. we worked on a shielded device allowing to directly recover the diffracted signal in a reflective configuration. Although this configuration is technically more complex to set up than the transmission one in terms of alignment and pump-probe synchronization, as it requires a perfect thickness characterization of the main target, it has several advantages. First, it allows to get rid of the corona noise by inserting a shielding high- $\mathrm{Z}$ material directly in the main target, and thus theoretically to gain in signal-to-noise ratio on the detectors. In the same way, the reflection geometry prevents the 0th-order incident X-ray beam to saturate the detectors as it goes out of the diffractometer. Moreover, it enables us to study phase diagrams and dynamics of phase transitions with different compression dynamics like shock, multi-shock and ramps as we can avoid probing the whole depth of the sample by adapting the X-ray penetration depth through the X-ray energy. Finally, this technique also allows us to work with heavy samples thicker than few microns on more modest laser facilities, which would not be possible with a transmission 
configuration because it would require a too intense X-ray source. To the best of our knowledge, a single study has recently collected powder diffraction data in a similar configuration on the ORION laser facility, in order to study the elastic-plastic transition of vanadium [48]. However, this study was carried out at relatively modest pressures, up to $70 \mathrm{GPa}$, and highlighted the difficulties in determining the precise positions of the diffraction lines at such pressures because of the increase in their width and of the diffuse background signal.

In the following, we present the ultrafast X-ray diffraction platform developed at LULI2000 laser facility using reflection geometry and which is also being studied to be used on the Laser MegaJoule facility. In particular, we detail in Sec. 2 the overall experimental design (Sec. 2.1), the detailed characteristics of the X-ray diffractometer (Sec. 2.2) and of the generated X-ray sources (Sec. 2.3), as well as the performances that can be achieved with the diffraction setup (Sec. 2.4). The observation of the iron $\alpha-\varepsilon$ transition in both shock and rampcompressed iron is then presented in Sec. 3, demonstrating the capabilities of such an experimental platform.

\section{Experimental setup}

\subsection{Overall experimental design}

Experiments were performed at the LULI2000 laser facility at the Ecole Polytechnique. LULI2000 is a two independent green beam $(527 \mathrm{~nm})$ laser capable of delivering $500 \mathrm{~J} /$ beam with adjustable temporal pulse shaping between 0.5 and 15 ns. The global experimental scheme shown in Figure 2.a follows classical diffraction schemes previously exposed.

One of the long-pulse lasers was focused on the main target, which is directly mounted on the diffractometer, to launch a shock or ramp-compression wave, bringing the sample to the desired high pressures and temperatures. This beam was spatially smoothed using a hybrid phase plate producing a flat top focal spot of $1300 \mu \mathrm{m}$ diameter to remove large-scale intensity modulations and to obtain a uniform compression in the transversal dimension. The second, shorter, laser beam ( 0.5 to $1 \mathrm{~ns}$ pulse duration) illuminated backlighter targets of $\mathrm{V}, \mathrm{Fe}$ or $\mathrm{Cu}$ to create a quasi-monochromatic X-ray source of $\mathrm{He}_{\alpha}$ emission between 5 and $9 \mathrm{keV}$. The X-ray energy is chosen according to the studied material, as well as to the target geometry. The best irradiation conditions, in terms of spot size, intensity and backlighter configuration, will be discussed in Sec. 2.3. This emission was spectrally monitored by a Von Hamos-type spectrometer for each shot.

Two VISAR operating at $532 \mathrm{~nm}$ and $1064 \mathrm{~nm}$ were used to monitor the thermodynamic state of the sample. Depending on the target geometry and of the compression method used, this system can possibly measure the velocities of the compression in the sample, on its release, or via a window material, as described in Sec. 3 . Moreover, an SOP system can collect the time-resolved optical radiation emitted from rear side of the studied sample. The short acquisition time requires a sufficient high photon flux to exceed noise level and thus a minimum sample temperature, typically over a few thousand Kelvin. Temperatures can then be extracted thanks to an absolute calibration or by comparison to a calibrated standard, like $\alpha$-quartz.

As stated in the introduction, the main problem of an X-ray diffraction experiment performed on a laser facility is to observe diffracted signal from a homogeneously-compressed studied sample above the parasitic Xray background. This one is generated both by the expanding plasma of the backlighter target, by the corona emission of the main target and by the diffraction/scattering of all this radiation through different components of the experiment other than the sample.

In order to improve the signal-to-noise ratio of the experimental set-up, as detailed in the following section, a specific strategy was adopted. We first focused on improving the diffraction signal from uncompressed materials and eliminating the maximum amount of spurious radiation by improving the device geometry, alignment, shielding, and optimizing the X-ray source. We then evaluated the additional noise introduced into the device 
during the compression of the main target and shielded the target and its support to reduce it as much as possible. Note that the level of this noise differs depending on the composition of the main target and the compression technique used. The results of these studies are presented in the following of this article.

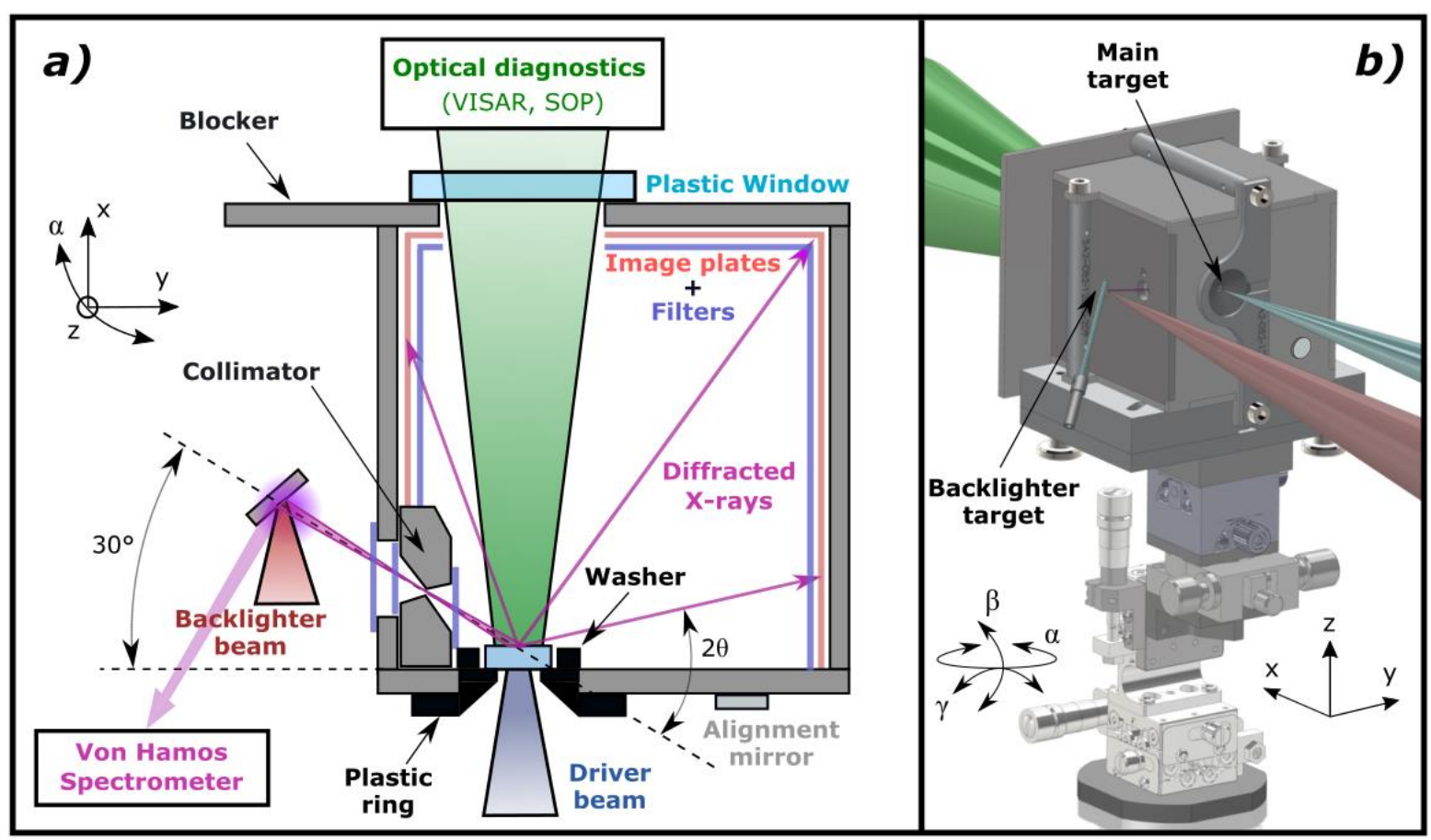

Figure 2: a) Schematic cross-section of the X-ray diffraction setup at LULI2000 laser facility in reflection geometry. The incident plasma-based ultrafast X-ray He $\mathrm{H}_{\alpha}$ emission is generated by interaction of the backlighter beam with a V, Fe or Cu foil, spatially filtered by a WC collimator and characterized by a Van Hamos-type spectrometer. The driver beam produces the dynamic compression. The diffracted X-rays with $2 \theta$ ranging between $30^{\circ}$ and $150^{\circ}$ are collected on five image plates (IP) enclosed into a WC-shielded box. Hydrodynamic conditions are measured through typical optical diagnostic (Doppler interferometry and pyrometry). b) 3D CAD view of the diffractometer, which is aligned in the LULI2000 experimental chamber thanks to a classical three axes / three angles positioner.

\subsection{Diffraction in reflection geometry}

The chosen reflection geometry allows a minimum collection $2 \theta$-angle of $30^{\circ}$, which corresponds to the angle between the direct X-ray axis and the front side of the box. Indeed, the rear surface of the main target, supported by a plastic ring and enlarged in Figure 2.a for easier reading, is completely cleared laterally to prevent any X-ray diffracted signal coming from the sample support itself. Note that this angle is very close to the minimum angle of detection of the transmission diffractometer used on the OMEGA facility $\left(25^{\circ}\right)$, as the very bright 0 -order does not allow access to information below this angle [39, 40]. In addition, the reflection geometry gives access to larger diffraction angles $2 \theta$ than can be achieved with the transmission configuration. In principle, it allows the observation of diffracted signals up to $150^{\circ}$ without being hindered by the collimator structure, and could even go as high as $170^{\circ}$ by reducing its size.

For external X-ray shielding, but also for robustness, the entire external structure of the diffraction box is made of $2 \mathrm{~mm}$ tungsten carbide (WC). In order to prevent any light leakage, the six independent plates are interlocked by means of a system of grooves such that there is always at least $1 \mathrm{~mm}$ of material everywhere between the outside and the inside of the box. The box is mounted on a classic tri-axis and tri-angles system, as 
seen in Figure 2.b, and aligned with the backlighter using a laser diode to materialize the direct X-ray axis. As this alignment is delicate, it cannot be re-performed between each shot without reducing the repetition rate. Thus, it is necessary that the structure composed of the front face and the side face supporting the collimator, which are defining axis of the X-rays, is completely fixed during the entire experiment. To achieve this, we have ensured that the system of five detectors (image plates) and associated plastic filters, which are amorphous and produce no diffraction lines, is integrated into a second internal structure that is integrally attached to the rear face of the shielded box. It is only the rear side of the box that is removed between shots, like a drawer. For readability reasons, this internal structure is not shown in Fig. 2.a. Furthermore, the positioning of the diffraction box on its support is rechecked with an accuracy better than $0.1^{\circ}$ before each shot using a simple system consisting of a second laser diode, a fixed spatial reference and a mirror integrated throughout the campaign on the front face of the diffractometer, as shown in Figure 2.

The collimator is also made entirely of tungsten carbide. This single-block piece fits perfectly the internal shape of the case and is held on it with two short, non-through screws. This keeps a minimum thickness of $5 \mathrm{~mm}$ of material between the backlighter and the main target outside the hole. Three double-cone collimators, such as the one shown in Figure 2.a, were used in our experiments, with holes of diameters 300, 350 and $550 \mu \mathrm{m}$ respectively, depending on the desired angular broadening, on the diffraction efficiency of the study sample, and consequently on the size of the area of the main target to be probed. In this configuration, this one is directly related to the magnification factor correlating the distances \{sample-collimator\}, close to $10 \mathrm{~mm}$, and \{backlightercollimator\}, which can be chosen and was typically $10-20 \mathrm{~mm}$ in our experiment. This geometry has been developed to avoid as much as possible that X-rays originating directly from the backlighter diffract on the collimator itself, compared to more classical pinholes tested in our first experiments.

Unprotected, a plasma plume created by the interaction of the backlighter beam with the backlighter sample penetrates into the diffractometer through the collimator. This plasma, still hot, then generates parasitic soft X-rays on the detectors, since the detectors integrate temporally the entire signal they receive during the whole experiment. To avoid this, a system of three successive filters made of $100 \mu \mathrm{m}$ of black plastic is arranged upstream and downstream of the collimator. The same applies to the plasma expanding outside around the box: this one generates radiation penetrating through the hole on the rear side designed for the visible diagnostics. Two countermeasures have been adopted to cut off this radiation completely, as shown in Figure 2.a: a blocker aligned with the rear side of the box physically stop the plasma expansion and a $4 \mathrm{~mm}$-thick optically transparent plastic window covers the entire hole absorbing soft $\mathrm{x}$-rays without restricting optical diagnostics.

Finally, in order to attenuate as much as possible the X-rays produced by the interaction of the driver beam with the main target, we have directly included in the latter a heavy material layer if the intrinsic absorption of the sample is not sufficient. Typically, a few microns of gold are sufficient for the different compressions tested on the LULI2000 facility and explained in Section 3. In addition, as mentioned before, we have positioned this target on a thick plastic ring, very easily repositioned between shots. The internal hole of the cone has a diameter of $2 \mathrm{~mm}$, and the minimum material thickness at the edge of the hole is $350 \mu \mathrm{m}$. The use of such a pinhole allows to filter efficiently (transmission coefficient $<10^{-3}$ ) soft X-rays up to more than $5 \mathrm{keV}$, while avoiding to generate additional radiation. At last, to ensure that no radiation can pass laterally if the first microns of the target are transparent to soft X-rays, an additional plastic washer, similar in height to the target, surrounds the target.

\subsection{X-ray source}

The geometry of our diffractometer allows studying the phases of crystalline materials with the DebyeSherrer method [3-5]. The X-ray source associated with this technique must then have sufficiently short emission 
time and small source size, as well as be as bright and as monochromatic as possible. Indeed, a spatially or spectrally too wide X-ray source reduces the angular resolution of the diffractometer, and even adds a parasitic diffraction signal. Moreover, an intense and badly filtered continuum X-ray radiation background associated with a dim source leads to a decrease in the signal-to-noise ratio, and consequently to difficulties in discerning the less intense diffraction lines.

On laser facilities, two processes for generating X-rays can be used to meet these specifications: $\mathrm{K}_{\alpha}$ and $\mathrm{He}_{\alpha}$ radiation, produced by the interaction of a short and intense laser with a metallic target, whose atomic number defines the radiated wavelength [22-25].

The first process, based on collisional ionization of the K-shell of the target material followed by radiative de-excitation, requires very high intensities to generate a large number of energetic electrons propagating into cold sample layers, and is achieved by using picosecond lasers. It thus has the advantage of being very short in time compared to the compression time of the studied samples. Nevertheless, as presented in previous studies, the use of an intermediate-size laser, such as PICO2000, does not generate a source bright enough to obtain a diffracted signal that can be well exploited on polycrystalline materials [36]. However, the use of a more powerful laser, such as PETAL or ARC, would meet this need [49].

Consequently, we have chosen the second process, i.e. $\mathrm{He}_{\alpha}$ emission, which generates a higher number of photons for the same X-ray energy [50]. This approach is similar to what is done in other diffraction experiments mentioned in the introduction. For this purpose, we focused a ns-laser pulse ( 0.5 to $1.0 \mathrm{~ns})$, capable of delivering $500 \mathrm{~J}$ at $527 \mathrm{~nm}$ onto the backlighter target, on a focal spot of few tens of $\mu \mathrm{m}$ in diameter and thus obtain intensities up to few $10^{16} \mathrm{~W} / \mathrm{cm}^{2}$. The interaction produces a hot ablation plasma where atoms are ionized to a He-like state with two bound electrons and are excited by collisions. The excited states relax through radiative emission, called $\mathrm{He}_{\alpha}$ radiation. In such a process, temporal emission of X-rays closely follows that of the laser pulse [23].

As in any diffraction experiment, the choice of the wavelength, and therefore of the backlighter material, must take into account the detectability of the lattice d-spacings over the detection range $\left[30^{\circ}-150^{\circ}\right]$. This then implies using typical energies from a few $\mathrm{keV}$ up to ten $\mathrm{keV}$. Typically, in most previous studies, $\mathrm{X}$-ray sources have been produced by irradiation of $\mathrm{Cu}, \mathrm{Fe}$ or Ge foils, respectively leading to complex $\mathrm{He}_{\alpha}$ emission centered around 8.4, 6.7, and $10.2 \mathrm{keV}$. A detailled characterization of these laser-based plasma sources at Omega laser facility with $\mathrm{I} \sim 10^{14}-10^{16} \mathrm{~W} / \mathrm{cm}^{2}$ have been recently reported in [25]. However, unlike the transmission configuration, our precise choice of the X-ray source wavelength is not motivated by the fact that a non-negligible part of the X-ray source must pass through the entire sample. On the contrary, it allows us to choose the thickness of the material of interest that we wish to probe, taking into account the absorption of the window material if it exists. It is then possible to diffract from a very thin layer of the sample in order to increase the homogeneity of the probed conditions, if this material diffracts sufficiently. On the other hand, if the material diffracts poorly, it will then be judicious to increase the penetration length. Note that these last remarks also apply to the transverse dimension and can help to choose the adequate size of collimator.

At LULI2000 facility, we have mainly worked with V, Fe and $\mathrm{Cu}$ backlighters, with the aim of generating $\mathrm{X}$-ray sources close to 5.2, 6.7 and $8.4 \mathrm{keV}$ respectively, which we consider interesting to probe a large number of different materials with different experimental strategies. The example of a typical X-ray spectra obtained by the interaction of the intense laser LULI2000 $\left(\mathrm{I} \approx 2.10^{15} \mathrm{~W} / \mathrm{cm}^{2}\right)$ with Fe and V backlighter targets and measured with the Von Hamos spectrometer, whose spectral resolution is close to $5 \mathrm{eV}$, are presented in Figure 3.a and 3.b respectively. It is notable that the emission spectra are largely dominated by the $\mathrm{He}_{\alpha}$ emission and presents similar intensity ratios between the different emission lines as those obtained at the Omega facility for comparable irradiation intensities in the case of iron [25]. From gaussian fits to the measured $\mathrm{He}_{\alpha}$ emission of $\mathrm{Fe}$ and V, we obtained $\mathrm{He}_{\alpha}$ energies of $6680 \mathrm{eV}$ (FWHM of $74 \mathrm{eV}$ ) for Fe and $5185 \mathrm{eV}$ (FWHM of $58 \mathrm{eV}$ ) for V. However, even 
by reducing their relative intensities by modifying the laser intensity, we could not remove the presence of other lines (mainly $\mathrm{K}_{\alpha}$ and $\mathrm{Ly}_{\alpha}$ ). Moreover, our spectrometer could not monitor more energetic lines (mainly $\mathrm{He}_{\beta}, \mathrm{He}_{\gamma}$ and $\mathrm{He}_{\delta}$ that present the highest relative intensities after the $\mathrm{He}_{\alpha}$ emission). The advantages and disadvantages of these annexed emission lines on the spatial calibration and resolution of the diffractometer are discussed in the following section.

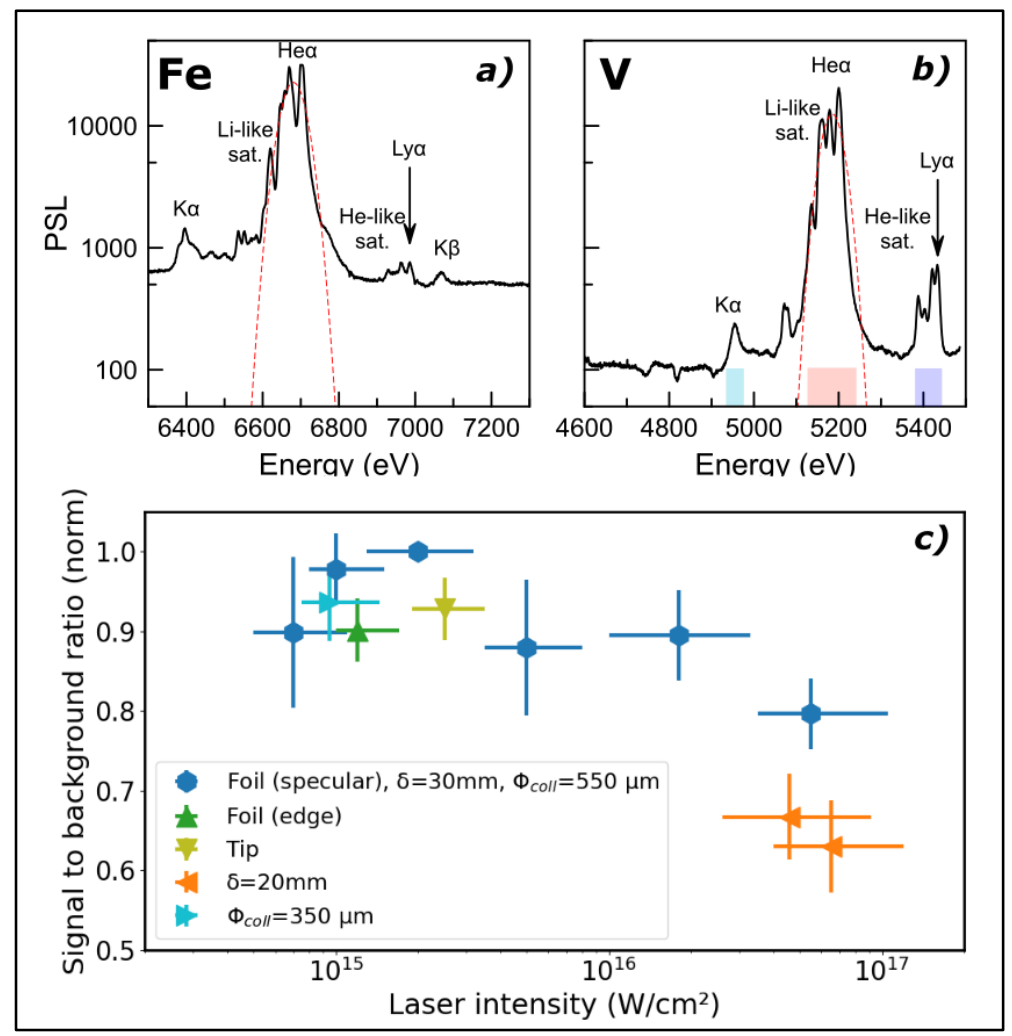

Figure 3: Spectrometer data for typical shot using $a \mathrm{Fe}(a)$ and $V(b)$ foil as backlighter. The emission spectra are largely dominated by the He $e_{\alpha}$ emission and present the same intensity ratios between the different emission lines as those obtained at the Omega facility for comparable irradiation intensities in the case of iron [25]. The energy of the He complex is fitted by a gaussian whose maximum position is used as the dominant wavelength for the diffraction analysis. The corresponding Full Width of Half Maximum (FWHM) is used to evaluate the spectral resolution in section 2.4. c) Evaluation of the best irradiation conditions of the Fe backlighter to maximize the diffracted signal to background, by modifying the laser intensity, the shape and orientation of the backlighter, the collimator diameter $\left(\Phi_{\text {coll }}\right)$, as well as the distance of the backlighter from the main target $(\delta)$. The loss of SNR is evaluated in relation to the shot that gave the best results, i.e. at about $2.10^{15} \mathrm{~W} / \mathrm{cm}^{2}$.

We also sought to optimize the scattering efficiency of the experimental setup by modifying various parameters of the interaction, such as the laser intensity (focal spot size, energy, and pulse duration), the shape and orientation of the backlighter target, and the distance between the backlighter target and the main target. For this purpose, we evaluated the diffracted signal-to-background ratio of different diffraction lines of a polycrystalline undriven tin foil. The conclusions of this statistical study are shown in Figure 3.c, in the case of a Fe backlighter target. Each point presents this ratio normalized by the one corresponding to the best results, i.e. at about $2.10^{15}$ $\mathrm{W} / \mathrm{cm}^{2}$, and results from about twenty comparison points at different locations of the diffractometer, and thus on the different detectors. Error bars are used to judge the dispersion of these measurements. 
A number of clear conclusions emerge from this study. First, there is an optimized intensity reducing the diffracted signal-to-background, close to few $10^{15} \mathrm{~W} / \mathrm{cm}^{2}$. This value is not surprising since it seems to be very close to the intensity that maximizes the conversion efficiency of laser energy into K-band X-rays (about 2\%) in the case of iron $[24,25]$. It should therefore be adapted according to the chosen wavelength, since the peak conversion efficiency decreases with increasing atomic number. Second, it does not seem useful to orientate the edge of a foil, or even a wire, towards the collimator to improve the quality of the experimental set up: the use of a sheet positioned as shown in Figure 2, i.e. to orientate the specular direction towards the collimator, allowed to obtain the best signal to background ratio. Such a geometry is, moreover, easier to align. Third, increasing the distance between the X-ray source and the diffraction box reduces the intensity of the background noise. This could be explained by a lower X-ray re-emission of the diffraction box materials located very close to the source. With the alignment system available at the LULI2000 facility, the maximum accessible distance between the two targets is close to $30 \mathrm{~mm}$. This is the distance we have retained for our experiments. Finally, it should be noted that the use of either collimator (with diameter of 350 and $550 \mu \mathrm{m}$ tested here) does not alter the quality of the resulting diffracted signal.

\subsection{Diffractometer performances}

In this section, we evaluate the performance of the diffractometer and describe our analysis protocol. To illustrate this, we rely on the XRD performed on an undriven target composed of $5 \mu \mathrm{m}$ of bismuth followed by 20 $\mu \mathrm{m}$ of diamond, obtained with a $\mathrm{V}$ backlighter. This example constitutes a typical test for the diffractometer: bismuth at ambient condition has a trigonal structure (space group $R-3 m \# 166)$ resulting in many diffracting ( $h k l$ ) planes and diamond is standard window material in shock experiments with reasonable transmission at $5.2 \mathrm{keV}$. Moreover, the interaction of the intense laser with a V backlighter can produce an X-ray source with several emission lines, as seen in the previous section, which adds difficulties in the analysis. We have therefore characterized the performance of the diffractometer in non-optimal conditions.

\subsubsection{Detailed geometry and spatial dispersion}

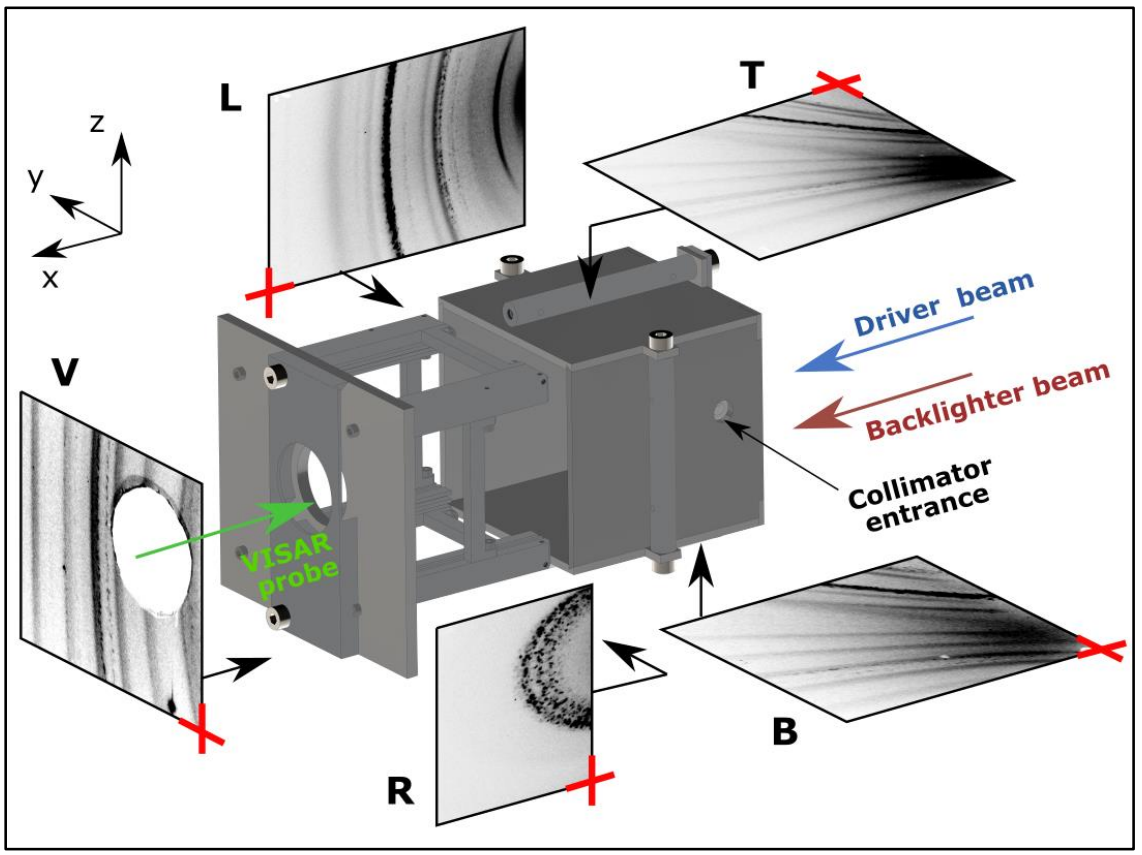


Figure 4: Diffracted raw data collected by the five image plates positioned inside the shielded diffraction box, obtained on an undriven Bi/Diamond target with a backlighter of Vanadium. The red crosses indicate the lower left corner of each IP as seen from the target. These IP are labelled (T)op, (B)ottom, (L)eft, (R)ight and (V)isar.

First, let us go back in detail to the geometry of the diffractometer. Figure 4 shows the position of the five detectors (IPs) placed on all sides of the box, except the target side, and they corresponding labels (L, T, B, V, R). The diffracted rays are collected between $2 \theta \approx 33^{\circ}$ and $152^{\circ}$ and $\phi$ ranging from $-95^{\circ}$ to $93^{\circ}$.

More precisely, as detailed in Figure 5, $\mathrm{L}$ collects the lowest diffraction angles $\left(2 \theta \approx\left[33^{\circ}, 88^{\circ}\right]\right)$ followed by $\mathrm{T}, \mathrm{B}, \mathrm{V}$ and $\mathrm{D}$ that respectively cover $2 \theta$-ranges of $\left[46^{\circ}, 128^{\circ}\right],\left[53^{\circ}, 123^{\circ}\right],\left[90^{\circ}, 132^{\circ}\right]$ and $\left[128^{\circ}, 152^{\circ}\right]$. In particular, in order to obtain two different powder signals on $\mathrm{T}$ and $\mathrm{B}$, these being located symmetrically on both sides of the $\mathrm{x}$-axis, we have deliberately distanced them differently from the diffracting sample. This vertical asymmetry allows to make a compromise between a higher signal for the closest IP (T) and a lower background noise for the furthest one (B).

The azimuthal coverage of the diffractometer ranges between less than $100^{\circ}$ at $2 \theta<50^{\circ}$ and $2 \theta>125^{\circ}$ to almost $180^{\circ}$ around $2 \theta \approx 105^{\circ}$. However, it should be noted that such coverage is smaller for small $2 \theta$ than what can be achieved in transmission geometry. This could be a limitation when studying textures or structural information strongly dependent on the azimuthal angle.

Finally, the intensity loss due to spatial dispersion is also shown in Figure 5 and is similar to the one observed on raw data: the intensity of diffracted X-rays remains constant along the z-axis for $\mathrm{L}, \mathrm{V}$ and $\mathrm{R}$, and clearly decreased by moving away from the target along the $\mathrm{x}$-axis. This is clearly visible on $\mathrm{T}$ and $\mathrm{B}$.

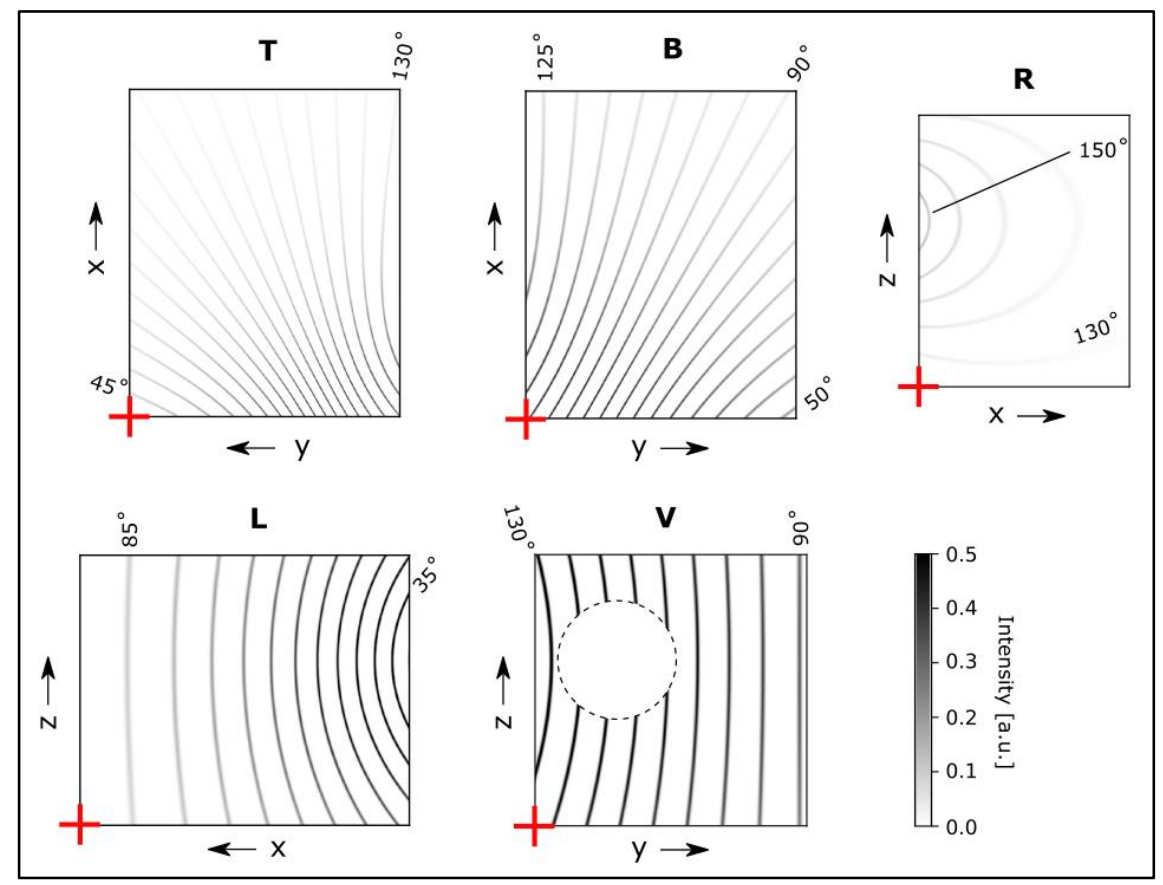

Figure 5: Effect of the spatial dispersion on each detector, oriented as seen from the target point of view. Each curve corresponds to the section of a diffraction cone coming from the centre of the sample with the detector. These diffraction cones have been simulated from an input 1d-spectrum composed of gaussian peaks separated by $5^{\circ}$ in $2 \theta$, with maximum intensities of 1 and FWHM of $0.2^{\circ}$. These last values have been arbitrary chosen for 
clarity. The corresponding $2 \theta$-values are given on each detector. The intensity loss due to spatial scattering is also shown in the figure and is similar to the one observed on raw data.

\subsubsection{Calibration, projection and correction}

The diffraction geometry, i.e. the transformation of the detector cartesian $(x, y)$ coordinates into the diffraction polar $(2 \theta, \phi)$ coordinates, is described using the formalism employed in the pyFAI package [51-53]. For a given wavelength, the position of one detector is defined by one distance, two coordinates in the detector's plane corresponding to the point of normal incidence of the X-ray source (PONIs), and three rotations. This Python package is developed by the European Synchrotron Research Facility and commonly used on synchrotrons, which allows the comparison of the diffraction data obtained on different facilities.

For a given detector, its size and its theoretical position in the diffractometer constrain the six calibration parameters. While this absolute description does not account for shot-by-shot variations of the experimental geometry, it allows defining acceptable ranges of values for the calibration parameters. These parameters are refined for each shot using internal calibration based on diffractions lines of undriven parts of the target. Then, refinement of the calibration, 2D-regrouping, i.e. projection of the images into the $(2 \theta, \phi)$ plane, and azimuthal integration are all performed with pyFAI, assuming a monochromatic X-ray source at the average energy of the $\mathrm{He}_{\alpha}$ emission ray of the corresponding backlighter.

Obtaining reference diffraction lines is then essential for a good calibration and different strategies allow to obtain this signal: 1) the transverse area probed by XRD is larger than the one compressed, which allows collecting diffraction lines from the uncompressed part of the sample; 2) efficient design of the pump-probe delay and consideration of the absorption length of the target allows probing both uncompressed and compressed material in time; 3) Multiple emission lines of the X-ray source can be advantageous if highly diffractive control materials are used, as it multiplies the number of usable calibration lines. The first option relies on a very good estimation of the sampling area, given further in the text. The second option is based on careful pump-probe timing and may require some preliminary shots. The third option adds difficulties in the analysis by adding diffracted lines coming from different X-ray energies. However, a simple trick allows transforming these additional lines back to the monochromatic framework of pyFAI: the diffraction lines of a calibrant's $(h k l)$ plane originating from other emission rays than the $\mathrm{He}_{\alpha}$ can be transformed into "fake $(h k l)$ planes" with apparent d-spacings at the $\mathrm{He}_{\alpha}$ wavelength. Such "fake" planes can then be added in calibration files used by pyFAI either by using the tabulated energies of the X-ray emission lines, or by using their energies measured directly with a spectrometer, or even indirectly via the diffractometer previously calibrated by a target whose constituent phases are well known. In the same manner, calibration files mixing planes belonging to different materials can be built (e.g. the sample and window material).

It is important to note that aforementioned calibrations are done in the same transversal plane, i.e. on the last $\mu \mathrm{m}$ of the studied target. This differs from transmission geometry experiments, which use the diffraction signal from thick partly compressed pinhole for calibration, and must consequently take into account of volume effects. However, in all cases, the design of the target and the nature of the backlighter should offer a reasonable number of calibration lines to minimize the overlap with the diffraction signal of interest.

After calibration, unwanted parts of the images are eventually masked and the intensity of each pixel is corrected for the solid angle dispersion discussed in the previous section. We do not correct the intensities for the efficiency variations of the IP due to the incidence angle of the incoming X-rays. Indeed, the maximum incidence angle in the present diffractometer is $\sim 72^{\circ}$, which results in negligible change in sensitivity of the IPs in the 5-10 
$\mathrm{keV}$ range considered here [40]. Finally, the images are projected into the $(2 \theta, \phi)$ plane and azimuthal integration is performed.

\subsubsection{Analysis illustration}

In the following, we apply the previous analysis to the complex case of the diffraction of multi emission line X-rays on a target composed of bismuth followed by a diamond window (raw images in Figure 4).

The result of this analysis is shown in Figure 6. The spatial calibration was performed by using the multiple diffraction lines from the (111) and (220) planes of diamond resulting from the polychromaticity of the V source (method 3). Diffraction lines from the diamond are easily recognizable as they present a granular aspect made by the individual spots from each crystallite that composes the window. The strongest lines associated with the $\mathrm{He}_{\alpha}$ emission (average wavelength measured at $2.3912 \AA$; see Figure 3) are marked by pale red areas in Figure 6 (the mean $2 \theta$-angles are shown as red lines). Additional lines associated with $\mathrm{He}_{\gamma}(1.9482 \AA), \mathrm{He}_{\beta}(2.0459 \AA)$ and $\mathrm{Ly}_{\alpha}$ $(2.2867 \AA)$ emissions are respectively shown as yellow, green and blue. The comparison of the integrated spectrum (lower panel in Figure 6) with the expected positions of the ( $h k l$ ) planes of $\mathrm{Bi}-\mathrm{I}$ at ambient conditions (solid blue lines, space group $R-3 m \# 166$ described considering rhombohedral axes [54]) validates the calibration procedure. We note that the $2 \theta$-position uncertainty induced by the calibration procedure depends on the number and the distribution of the calibration lines on a given detector. However, by repeating this procedure several times on the different detectors, we estimated the resulting uncertainty on $2 \theta$-angle is $\sim 0.15^{\circ}$. 


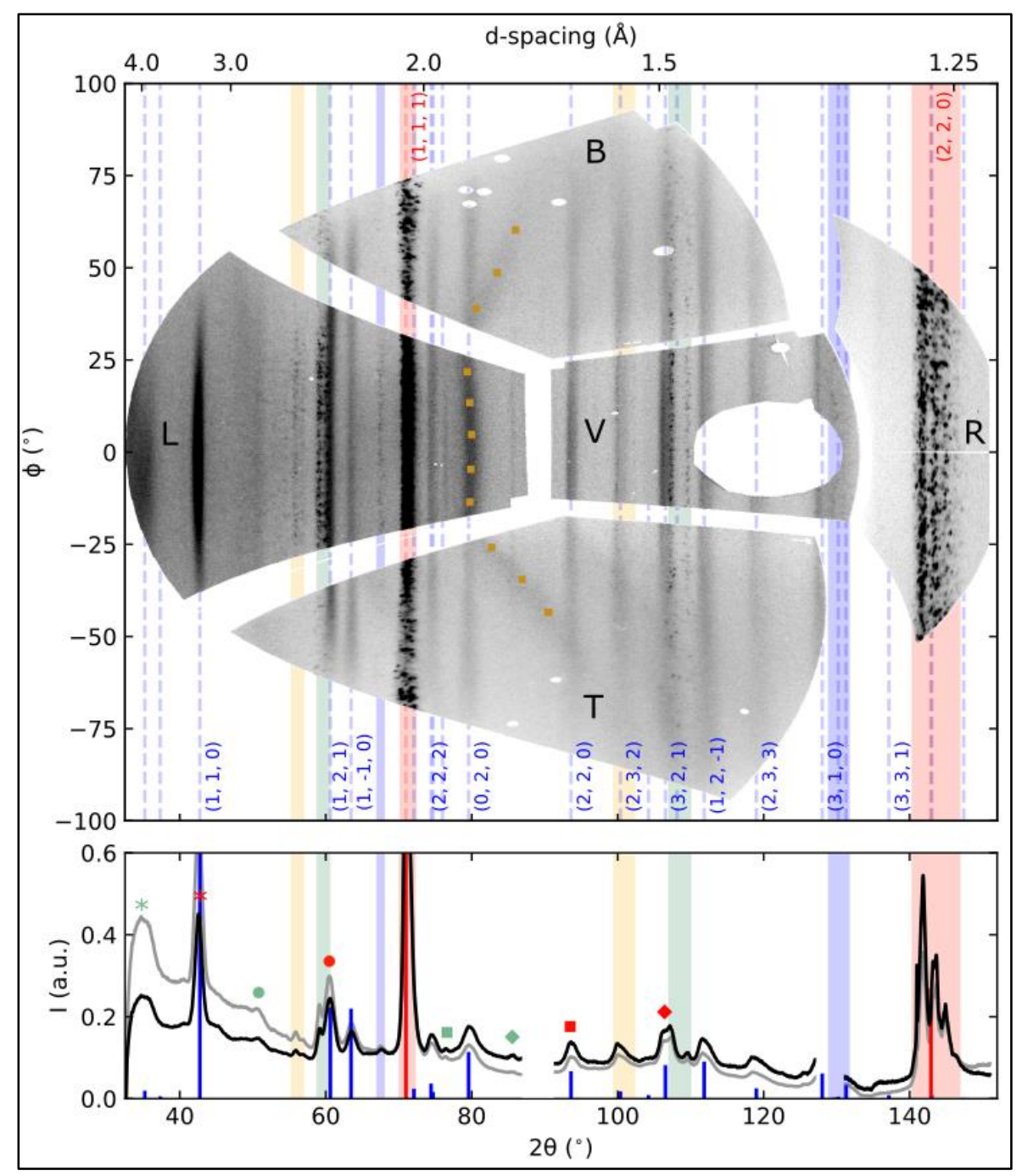

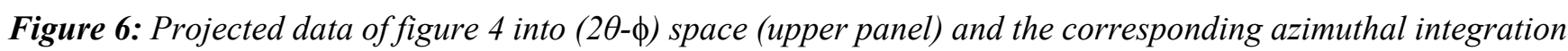
(lower panel). Theoretical diffraction lines from He source of $V$ for Bi-I and diamond at ambient conditions are shown in blue and red, respectively. Only the most intense Bi-I (hkl) planes have been labelled. The coloured areas show the position of the diamond diffraction lines and their colour refer to the corresponding emission line of $V$ (yellow: He; green: He ; blue: Ly $y_{\alpha}$; red: He $e_{a}$ ). Moreover, parasitic signal from WC collimator is shown by the orange squares. In the lower panel, the black and grey lines correspond to the azimuthally integrated spectra respectively with and without the solid angle correction of the intensities. The blue (red) vertical lines show the expected positions for the (hkl) planes of Bi-I (diamond) and their relative intensities, considering a monochromatic source at the V He wavelength. Some Bi-I (hkl) planes affected by the He $e_{\beta}$ of the V source are showed by green symbols. Each symbol refers to a considered (hkl) plane, which is marked with red symbols in the case of diffraction by He emission. Note that some point over- or under-intensities coming from the filters or the scanner have been masked prior integration, as it is visible on the upper panel. Moreover, to avoid any artificial peaks or discontinuities due to slight background differences, we have masked some interface regions between IPs on the integrated spectrum.

In this particular case, we note that the polychromaticity of the $\mathrm{X}$-ray source $\left(\mathrm{He}_{\beta}\right.$ emission) is also visible in the Bi-I diffraction signal as shown by the color symbols in Figure 6, each symbol being associated with a given $(h k l)$ plane of Bi-I. Such a weak diffracted signal could be suppressed with the help of filters placed in front of the 
IPs and composed of materials with carefully chosen absorption edges, since the energy of the $\mathrm{He}_{\beta}$ emission is much higher than that of the $\mathrm{He}_{\alpha}$ emission. However, these signals disappear as soon as the signal-to-noise ratio decreases. This is naturally the case when studying compressed materials.

Then, we notice that the solid angle correction of the pixel intensities has its largest effect below $2 \theta=60^{\circ}$ in the integrated spectrum (difference between the gray - no correction - and black - correction - curves in Figure $6)$.

Moreover, we note that additional broad diffraction lines with a different geometry appear on the detectors $\mathrm{T}$, $\mathrm{B}$ and $\mathrm{L}$ between $2 \theta \approx 80^{\circ}$ and $90^{\circ}$. This interfering signal, highlighted by orange squares in Figure 6 , comes from the diffraction of the WC collimator, and therefore a different source point from the sample. It will be eventually removed in the next versions of the diffractometer. In spite of this technical defect present on a restricted area of the diagnosis, this example demonstrates that it is possible to study multi- $\mu \mathrm{m}$ thick heavy materials such as bismuth on LULI2000-type laser facilities, which would not have been possible by using diffractometer with transmission geometry due to the X-ray opacity of such a heavy material at an X-ray energy allowing to correctly separate its numerous diffraction lines of interest.

Finally, we notice that the diffraction lines widen with increasing $2 \theta$-angles. This is clearly visible on the diamond calibration lines. This can be explained by the intrinsic instrumental broadening of our diffractometer, detailed in the following section.

\subsubsection{Instrumental broadening}

The total angular instrumental broadening $\sigma_{\text {instr }}$ of the diffractometer is limited by the non-infinitely fine spectrum of the X-ray source, by the non-finite size of the area probed by the X-ray source, as well as by the spatial resolution of the detectors. In the following, we always consider $\sigma$ as the FWHM of the different broadening functions.

The first component results from the spectral width of the considered emission rays, i.e. of the $\mathrm{He}_{\alpha}$ line in our case. This is essentially due to the multiple $\mathrm{He}_{\alpha}$ lines, which are typically separated by $0.5 \%$ in energy. More precisely, considering such a spectral width $\sigma_{\lambda}$ around a given wavelength $\lambda$, the spectral resolution has been detailed in [37] and can be approximated by:

$$
\sigma_{x-\text { ray }}(2 \theta, \lambda)=\frac{2 \sigma_{\lambda} \tan (\theta)}{\lambda}
$$

This results in an increasing broadening with increasing $2 \theta$, and becomes predominant with respect to the other effects mentioned above, especially above $\approx 80-100^{\circ}$. In the example presented in Figure 7 , the $\mathrm{V} \mathrm{He}-\alpha$ emission line at $\lambda=2.3912 \AA$ has a spectral width $\sigma_{\lambda} \approx 0.0267 \AA$ (see Figure 3), this results in a spectral broadening of $\sigma_{\text {x-ray }}=0.89^{\circ}$ for the (111) diffraction line of diamond at $2 \theta=70.73^{\circ}$ and $\sigma_{x \text {-ray }}=3.67^{\circ}$ for the (111) diffraction line of diamond at $2 \theta=141.89^{\circ}$. 


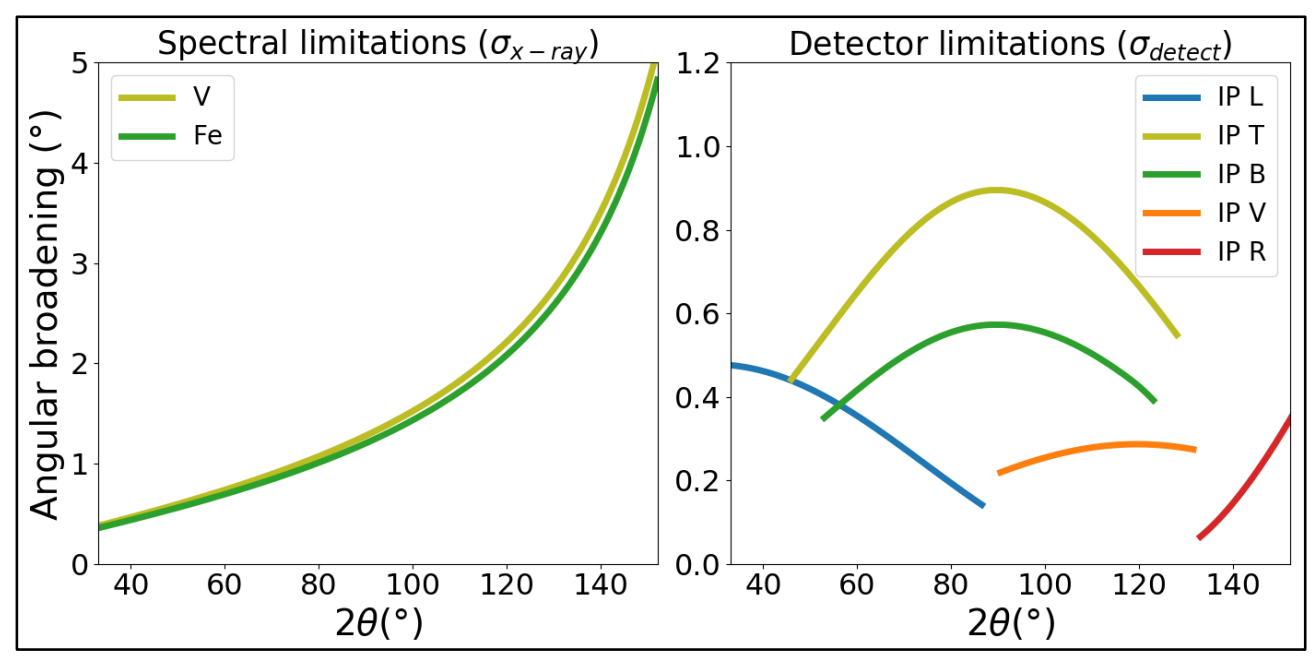

Figure 7: On the left: Angular broadenings due to the spectral width of the He- $\alpha$ emission lines, as a function of the diffraction angle $2 \theta$. Such an effect become the predominant effect for line-broadening at large $2 \theta$-angle. On the right: maximum angular broadening of each IP, as a function of the diffraction angle $2 \theta$. These values are not limiting in our experiments.

Regarding detectors, we used Fujifilm ${ }^{\mathrm{TM}}$ BAS-MS imaging plates (IP's), which were read with a Typhon ${ }^{\text {TM }}$ FLA 7000 scanner with a L5 dynamic range, a pixel size of $50 \mu \mathrm{m}$ and sensitivity S10000. We can consider that such detectors have a spatial resolution close to $250 \mu \mathrm{m}$ [29]. By 3D projection, we can then go back to the angular separation offered by each detector at each point, which makes it possible to translate more simply the separation of the iso-angle cone sections shown in Figure 5. The maximum angular broadening of each detector $\sigma_{\text {detect }}(2 \theta, \phi)$ is also shown in Figure 7 . The detector $\mathrm{T}$ is the closest to the target and therefore has the lowest angular resolution. However, the detector resolutions are still quite acceptable given the other spatial and spectral limitations of such experiments.

Finally, the angular broadening of our system is also limited by the size of the probed area, i.e. from the fact that diffracted signal comes from a finite volume and not from a source point, as the finite size X-ray source is sampled by a circular collimator of few hundred micrometers in diameter, resulting in an ellipsoidal spot on the sample. Since we are essentially probing the sample's surface, this angular broadening $\sigma_{\text {prob }}$ mainly depends on the angular aperture of the source $\mathrm{X}$ on this sample, which is not linked to the diffraction angle $2 \theta$ (as directly related to the diameter of the collimator, to its distances from the main target and from the backlighter target and to the spatial extent of the X-ray source), as well as on the distance between the sample and the detectors. However, in the case of the reflection configuration, and contrary to the transmission one, diffraction on a single $(h k l)$ plane has the effect of refocusing X-rays arriving on either side of the sample at slightly different incidence angles. Thus, when the dimensions of the diffraction box are not large enough to allow the re-defocusing of the X-ray beams, which is the case for our diffractometer, the maximum angular broadening due to the size of the probed area corresponds to the aperture angle of the X-ray source [40]. Typically, considering a maximum focal spot size of the intense laser on the backlighter target of $200 \mu \mathrm{m}$ in diameter, a collimator-to-sample distance of $10 \mathrm{~mm}$ and a collimator-to-backlighter distance of $20 \mathrm{~mm}$, the maximum theoretical geometric broadening of our instrument corresponds to $\sigma_{\text {prob,max }}=1.05^{\circ}, 1.20^{\circ}$ and $1.75^{\circ}$ using a collimator with a diameter of $300 \mu \mathrm{m}, 350 \mu \mathrm{m}$ and $550 \mu \mathrm{m}$ respectively. This limitation could be predominant at low angles of diffraction $2 \theta$. 
Thus, the total $2 \theta$ instrumental broadening $\sigma_{\text {instr }}$ is due to a convolution of these several independent broadening sources. When these different components can be well described by Gaussian distributions, we can treat this convolution as the quadrature sum of the various terms:

$$
\sigma_{\text {instr }}(2 \theta, \Phi)=\sqrt{\sigma_{x-r a y}^{2}(2 \theta)+\sigma_{\text {detect }}^{2}(2 \theta, \Phi)+\sigma_{\text {prob }}^{2}(2 \theta, \Phi)}
$$

To go further, we sought to evaluate experimentally the size of the surface area probed by X-rays on our studied samples, in order to validate in particular the correct alignment of our measuring instrument. To do this, we carried out, for the two smallest collimators, three distinct shots where we modified the geometry of the undriven sample, while taking care to keep similar backlighter irradiation conditions. The first sample corresponded to a sheet with dimensions much larger than that of the elliptic probed area, the second to a wellcentred rectangular target with dimensions similar to those of the theoretically probed area and the third to the counter shape of the second. From these series of shots it can be concluded that $80 \pm 5 \%$ of the diffracted signal comes from the theoretical probed area. By taking into account the diffractometer geometry and the position and size of the collimators, as described in Section 2.2, this result means that, in the worst case, the area actually probed can be shifted laterally by $220 \mu \mathrm{m}$ or vertically by $110 \mu \mathrm{m}$ with respect to the theoretical probed area.

\section{Results on different experimental strategies}

As previously mentioned, the reflection geometry of our diffractometer allows to probe a desired thickness of the rear side of the sample by choosing the wavelength of the X-ray source appropriately. It is then possible to probe the solid phases of compressed materials with different compression dynamics, even by using a relatively long probe, by considering different experimental strategies. As a proof-of-concept, we report direct structural measurements of the well-known $\alpha-\varepsilon$ transition in shock and ramp-compressed iron.

\subsection{Time-resolved shock compression}

\subsubsection{Experimental strategy}

The strategy used to study the phases of shock-compressed iron is the same as the one used a few years ago on the GEKKO XII and LULI2000 laser facilities with the analyzer crystal presented in the introduction [36]. The idea is to probe, by adequately adjusting the wavelength of the X-ray source and the pump-probe delay, the last microns of the sample of interest before the shock passes through it. This then allows to probe the sample both at ambient conditions, providing a simple calibration reference to the diffractometer, and at homogeneously shockcompressed conditions, despite the low temporal resolution. Indeed, in that case, the main contribution of the diffracted signal will come directly from the shock front and not from inside the compressed target which may already be starting to expand.

For this, the main target, which must be perfectly characterized in terms of thickness, is here composed of an ablator of polypropylene, about $30 \mu \mathrm{m}$, glued on an iron foil. The thickness of this foil must be high enough to filter the soft X-radiation coming from the plasma corona generated on the front face by the interaction of the ablator with a laser with a square time profile, such as the one shown in Figure 8.a. Experimentally, we determined that about $50 \mu \mathrm{m}$ of iron could perfectly achieve this shielding for laser intensities up to $2.10^{13} \mathrm{~W} / \mathrm{cm}^{2}$.

The shock generated by this laser-plasma interaction then propagates inside the target and compresses the iron along its Hugoniot. The shock breakout time and the velocity of the iron free surface are monitored by the optical diagnostics VISAR, as shown in figure $8 . \mathrm{b}$ (solid curves), with sensitivities of 4.96 and $3.43 \mathrm{~km} / \mathrm{s} /$ fringe at 532 and $1064 \mathrm{~nm}$ respectively. As we measure directly the fluid velocity [55], these two parameters allow us, via 
a hydrodynamic simulation (dashed curves), to evaluate the speed of the shock passing through the iron, and thus to determine its pressure and density, in particular at the moment when the iron is probed. We performed such simulations with the 1D Lagrangian hydrodynamic ESTHER code developed by CEA [56], using the SESAME 2150 equation of state [57]. The simulated density map of this example is shown in Figure 8.c. and allows evaluating the hydrodynamic conditions probed between 6.7 and $7.2 \mathrm{~ns}$, i.e. in the time interval during which Xray emission was generated by the interaction of the intense laser beam with a backlighter target of iron.

In that case, the diffracted X-rays collected by IPs came mostly from the first microns of the iron rear side due to X-ray absorption in iron, as shown in Figure 8.d. Indeed, $80 \%$ of the X-rays are already absorbed in the first $8 \mu \mathrm{m}$ of iron on a round trip, considering their angle of incidence of $60^{\circ}$ relative to the target normal. Thereby, the vast majority of the diffracted signal comes from the black space-time rectangle shown in Figure 8.c., i.e. from uncompressed cold iron and highly compressed iron. We have thus used these simulations to estimate the probed thermodynamic conditions, by evaluating diffracted signal coming from various depths in iron over the entire duration of the X-ray probing, taking into account its absorption. This then allows us to estimate the density and the pressure of the probed shocked iron at $11.58 \pm 0.36 \mathrm{~g} / \mathrm{cm}^{3}$ and $183 \pm 23 \mathrm{GPa}$. The temporal and spatial uniformity of the sample density and pressure during X-ray exposure is evaluated based on the variance of the density and stress values in this region.

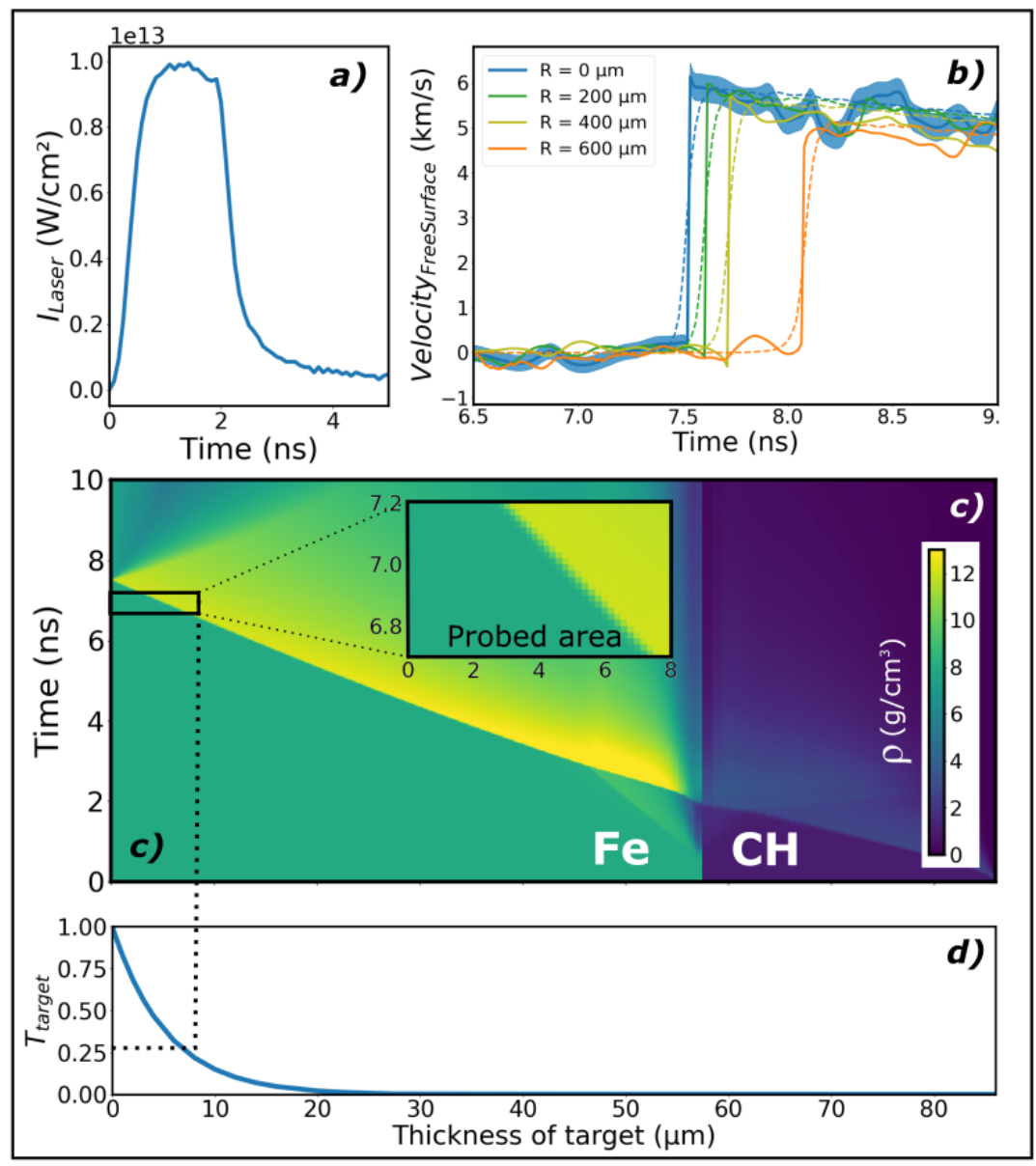

Figure 8: a) Temporal profile of the driver beam used to generate the shock. b) Calculation (dotted line) and measurement (solid line) of the iron free surface velocity as a function of time using VISAR diagnosis, at distance $R$ from the centre of the sample. The blue band represents the measurement uncertainty in determining the phase of the VISAR fringes (about 5\% on a fringe). c) Density map obtained by means of a hydrodynamic simulation carried out with the ESTHER code (laser comes from the right) and allowing to highlight the hydrodynamic 
conditions surveyed, both cold $(\approx 70 \%)$ and homogeneously compressed $(\approx 30 \%)$. d) Two-way X-ray absorption of the sample at $6.70 \mathrm{keV}$, considering an angle of incidence of $60^{\circ}$.

It is important to note that this hydrodynamic estimation has been carried out by also taking into account the transverse compression heterogeneities. For this shot, we used the $350-\mu \mathrm{m}$ diameter collimator to probe a $1050-\mu \mathrm{m}$ wide and $525-\mu \mathrm{m}$ high area. Based on the results presented in Sec. 2, we integrated the hydrodynamic conditions over a slightly larger area, i.e. close to 1200 x $600 \mu \mathrm{m}$. For this purpose, we have therefore achieved several hydrodynamic simulations corresponding to the VISAR measurements carried out over the entire probed area, as shown in Figure 8.b. Typically, to find the experimental results measured at $200 \mu \mathrm{m}, 400 \mu \mathrm{m}$ and $600 \mu \mathrm{m}$ from the center of the probed zone, an intensity reduction factor of $5 \%, 10 \%$ and $25 \%$ respectively had to be applied to the hydrodynamic simulations. These differences are the result of a slight curvature of the shock wave. However, the transverse hydrodynamic conditions remain very similar over most of the area probed: a difference of less than $0.1 \mathrm{~g} / \mathrm{cm}^{3}$ is typically predicted along the shock front between the focal spot centre and an area located $400 \mu \mathrm{m}$ away. Moreover, the contribution of the diffracted signal at the extremities of the probed zone remains marginal, due to the elliptical nature of the probed zone and the fact that the shock, whose velocity is decreasing at the edge of the focal spot, is located a little deeper in the sample at the probed time.

\subsubsection{Diffraction results}

Figure 9 shows the diffraction signal associated with the results presented above. Strong diffraction lines of ambient bcc $\mathrm{Fe}$, corresponding to the $\mathrm{He}_{\alpha}$ emission line, are logically present on the images and have been used to calibrate the experimental geometry. Three diffraction lines from shocked Fe are visible from $2 \theta=55^{\circ}$ to $2 \theta=$ $65^{\circ}$ and corresponds to hep Fe (100), (002) and (101) planes.

In this particular case, the parasitic signal from WC diffraction originating from the collimator (see section 2.4.3 and orange dotted line in Figure 9) unfortunately overlaps the (101) diffraction line from hop Fe between $\phi$ $=-40^{\circ}$ and $\phi=40^{\circ}$, mainly on IPs L and T. For this reason, we do not perform a large azimuthal integration over all images but we integrate on a restrictive 20- $\phi$ region, as shown in Figure 9, where the contribution from WC is separated from Fe diffraction lines.

After azimuthal integration, we fit a baseline to correct the diffraction signal for large amplitude background intensity variations. In this present case, we use a cubic spline, as seen in Figure 9.b. This choice is somewhat arbitrary but we found that it induces negligible variations (much smaller than $2 \theta=0.1^{\circ}$ ) in the positions of peak maxima if a reasonably small number of spline nodes is used (4 nodes in the present case). Thus, we estimate that the calibration procedure (see 2.4.3) and the choice of the background result in an uncertainty of $\sim 0.15^{\circ}$ on $2 \theta$. This accuracy is similar to the one obtained with the diffractometers PXRDIP and TARDIS, working in transmission geometry and quoted in introduction [39,40]. We then fit each spectrum by a sum of Gaussian functions. Table 1 shows the positions in $2 \theta$ and d-spacing of maxima for each $(h k l)$ plane from the least-square fitting procedure, as well as the FWHM of the corresponding Gaussian curves in $2 \theta$-angle $\left(\sigma_{g}\right)$. This other procedure returns an uncertainty on the $2 \theta$-position of each diffraction peak. The total uncertainty on the position of each peak (shown in Table 1) results from the convolution of the two errors described above. Thus, for welldefined peaks, 20-positions are obtained with a typical error of $0.15-0.17^{\circ}$, as mentioned above, whereas for illdefined peaks larger errors arise, e.g. $0.72^{\circ}$ for Fe hcp (100) peak due to its proximity with the intense Fe bcc (110)

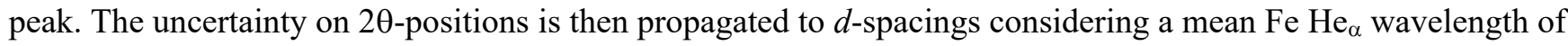
$1.8561 \pm 0.002 \AA$. This results in an average uncertainty of $0.2-0.3 \%$ on $d$-spacings when peaks are well defined. 


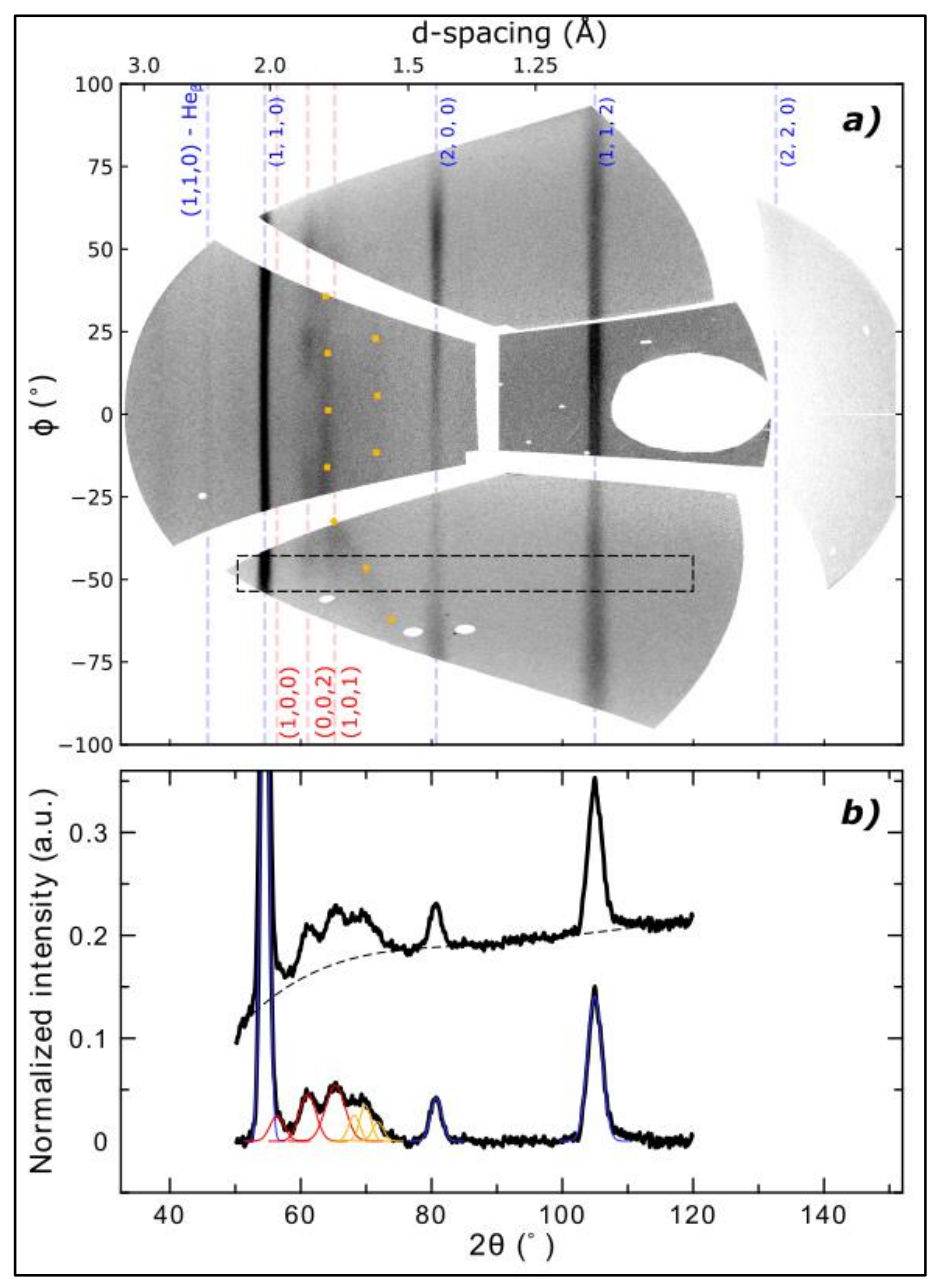

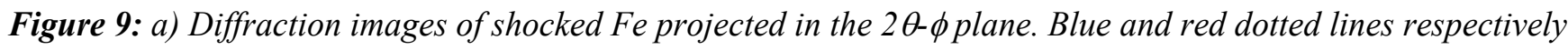
indicate the $2 \theta$ positions of bcc Fe at ambient conditions and shock-compressed hcp Fe. Black dashed lines delimit the area in which azimuthal integration has been performed in order to avoid overlapping between hcp Fe (101) diffraction line and the spurious signal from WC collimator shown by the orange dotted line. b) XRD spectrum corresponding to the integration area with its corresponding spline background. The spectrum after background subtraction is shown together with Gaussian curves fitting bcc Fe (blue), hcp Fe (red) and the parasitic signal from $W C$ (orange). Finally, we note that two broad and faint lines at around $38^{\circ}$ and $50^{\circ}$ are in the background signal, as also visible with undriven Fe target, but their origin is still not identified.

\begin{tabular}{|c|lllll|}
\hline Plane & $2 \theta\left({ }^{\circ}\right)$ & $d(\AA)$ & $\sigma_{g}\left({ }^{\circ}\right)$ & $\sigma_{2 \text { instru }}\left({ }^{\circ}\right)$ & $\sigma_{2 \text { einstruthydro }}\left({ }^{\circ}\right)$ \\
\hline BCC & & & &
\end{tabular}




\begin{tabular}{|l|lllll|}
$(110)$ & $54.49(15)$ & $2.027(6)$ & 1.42 & 1.40 & 1.40 \\
$(200)$ & $80.63(17)$ & $1.434(3)$ & 2.08 & 1.94 & 1.94 \\
$(211)$ & $105.02(15)$ & $1.170(2)$ & 2.60 & 2.69 & 2.69 \\
\hline HCP & & & & & \\
$(100)$ & $56.08(72)$ & $1.975(23)$ & 2.88 & 1.42 & 2.78 \\
$(002)$ & $61.08(17)$ & $1.826(5)$ & 2.99 & 1.52 & 3.04 \\
$(101)$ & $65.29(16)$ & $1.720(4)$ & 3.56 & 1.60 & 3.25 \\
\hline
\end{tabular}

Table 1: $2 \theta$-angles of peak maxima, corresponding $d$-spacings, and measured FWHM $\left(\sigma_{g}\right)$ of the bcc and hcp Fe (hkl) planes. The measured broadening $\sigma_{g}$ is compared to the instrumental broadening $\left(\sigma_{2} \theta_{i n s t r u}\right)$ and the expected total broadening $\left(\sigma_{2}\right.$ ainstru+hydro $)$ which includes the hydrodynamic dispersion obtained from the hydrodynamic simulation presented in section 3.1.1. Values in parenthesis give the error on the last digit.

$d$-spacings and their associated errors $\sigma_{d}$ are then used to determine the cell parameters. In the case of bcc and hcp iron we are interested in, i.e. for cubic and hexagonal crystalline systems, $d$ is related to the cell parameters by equations (3) and (4) respectively.

$$
\begin{gathered}
d=\frac{a}{\sqrt{h^{2}+k^{2}+l^{2}}} \\
d=\frac{a}{\sqrt{\frac{4}{3}\left(h^{2}+h k+k^{2}\right)+\left(\frac{a}{c}\right)^{2} l^{2}}}
\end{gathered}
$$

The cell parameters ( $a$ for the cubic system; $a$ and $c / a$ for the hexagonal system) are then fitted using a least-square procedure by minimizing $\chi^{2}$ :

$$
\chi^{2}=\sum_{i=1}^{N} \frac{\left(d_{i}-d\right)^{2}}{\sigma_{d, i}{ }^{2}}
$$

where $N$ is the number of $(h k l)$ planes considered, $d_{i}$ and $\sigma_{d, i}$ are the corresponding d-spacings and errors, and $d$ the d-spacing computed for a given set of cell parameters using equation (1) or (2) for bcc or hcp Fe. Similar approach is described in Polsin's PhD thesis [45].

For unshocked bcc Fe, we obtain $a_{b c c}=2.867 \pm 0.002 \AA$ which results in a unit-cell volume $V_{b c c}=23.558$ $\pm 0.043 \AA^{3}$ and a density $\rho_{b c c}=7.873 \pm 0.014 \mathrm{~g} / \mathrm{cm}^{3}$. Evidently, we retrieve the expected values of $a=2.8664 \AA$ and $\rho=7.8753 \mathrm{~g} / \mathrm{cm}^{3}$ as the diffraction lines from bcc Fe have been used to calibrate the experimental geometry. The errors associated with $a_{b c c}, V_{b c c}$, and $\rho_{b c c}$ are representative of the intrinsic accuracy of the diffractometer.

For hcp Fe, we obtain $a_{h c p}=2.253 \pm 0.007 \AA$ and $c / a=1.621 \pm 0.007$ which results in $V_{h c p}=16.051 \pm$ $0.161 \AA^{3}$ and $\rho_{h c p}=11.556 \pm 0.116 \mathrm{~g} / \mathrm{cm}^{3}$. Therefore, the mean density of hcp Fe measured by XRD is very close the one obtained in the mean value estimated from the hydrodynamic simulation $\left(11.58 \mathrm{~g} / \mathrm{cm}^{3}\right)$. This example shows that the present diffractometer allows to probe the structure shocked materials and their density with an accuracy of $\sim 1 \%$. Note that this measurement is consistent with previous measurements carried out on shock- 
compressed iron, which have shown the presence of the hcp phase close to such densities and pressures using Xray diffraction [36] or XAFS spectroscopy [58, 59].

Moreover, as shown by $\sigma_{g}$ in Table 1, diffraction peaks from bcc Fe presents typical broadening as expected from the instrumental response (cf. section 2.4.4). On the contrary, peaks from Fe hcp present an additional broadening that may result from the hydrodynamic dispersion of the probed conditions, or by other effects intrinsic to the hcp phase (small grain size ...). We estimate the total $2 \theta$-broadening $\left(\sigma_{2 \theta \text {,instru+hydro }}\right)$ caused by the density dispersion predicted from the hydrodynamic simulation $\left(\sigma_{\rho}=0.72 \mathrm{~g} / \mathrm{cm}^{3}\right)$, taking into account the instrumental response $\left(\sigma_{2 \theta \text {,instru}}\right)$. Assuming Gaussian distributions of the dispersions, the convolution of all broadening terms results in:

$$
\sigma_{2 \theta, \text { instru+hydro }}=\sqrt{\sigma_{2 \theta, \text { hydro }}^{2}+\sigma_{2 \theta, \text { instru }}^{2}}
$$

Considering an isotropic compression and the Bragg's law [40], small variations in density can be expressed as function of $2 \theta$ as:

$$
\sigma_{2 \theta, \text { hydro }}=\frac{2 \sigma_{\rho}}{3 \rho} \tan \theta
$$

The resulting broadening $\sigma_{2 \theta \text {,instru+hydro }}$ expected for each $(h k l)$ plane is given in Table 1 and shows a good agreement with the measured broadening $\sigma_{g}$, indicating that line broadening is dominated by density inhomogeneities in the shocked material.

\subsection{Time-resolved ramp compression}

\subsubsection{Experimental strategy}

In order to cover a larger part of the dynamic phase diagram of materials of interest, it is also very interesting to compress these materials in a smoother way, for example using ramp-shaped laser temporal profiles. This allows to move away from their respective Hugoniot, reaching lower temperatures for similar pressures, and to remain in a solid state at pressures of up to hundreds of GPa without crossing their melting curve.

In contrast to shock compression, the thermodynamic state of a ramp-compressed material changes gradually over time and through the thickness of the target, with a characteristic time similar to the pulse length of the drive laser. Probing this material in a single state without being hindered by the inherent density gradients is therefore complex. The reflection configuration of our diffractometer then allows two strategies to be used to probe homogeneous hydrodynamic states.

The first possible strategy consists in probing the very last microns of the material of interest in a very short time compared to the time of evolution of its hydrodynamic conditions, i.e. of the order of picosecond. This strategy has a big advantage: it allows choosing the compression velocity of the sample, by choosing the right ramp/target thickness combination, and to approach, for example, the quasi-isentropic conditions of the material. However, it requires the use of a sufficiently intense $\mathrm{K}_{\alpha}$ picosecond X-ray probe, which we do not have on the LULI2000 laser system, as discussed in section 2.2. It could however be used on a LMJ-PETAL type facility.

With a nanosecond X-ray probe, there is no other possibility than to remain stable during the entire probe time. For this purpose, a strategy that has been commonly used on OMEGA and NIF facilities for some years now has been successfully employed: the sample of interest is inserted into a diamond anvil in order to generate a succession of compressing wave reverberations at the diamond/sample interfaces. By carefully and judiciously 
choosing the association \{temporal laser pulse shape, ablator, thickness of the different layers\}, these reverberations can drive pressure uniformity within the sample for several nanoseconds. Typically, the sample must be relatively thin to ensure a sufficient number of reverberations to reach pressure equilibrium but thick enough to diffract enough signals. In addition, the diamond window must be thick enough to prevent its expansion from releasing the pressure too early in the sample, i.e. before the compression peak is reached. On the other hand, it must be thin enough to avoid generating too much stray diffracted signal, and to prevent the formation of shocks that can complicate the interpretation of the VISAR data, necessary to trace back the compression history of the various target materials. It should also be noted that a thin layer of shielding material can be inserted into the target to protect detectors from corona X-rays.

For this example, we then used the target, as described in Figure 10:8 $8 \mathrm{~m}$ of iron were surrounded by two diamonds. The first diamond, $23 \mu \mathrm{m}$ thick, is used as an ablator. The second diamond, $23 \mu \mathrm{m}$ thick, forms the window. A $2 \mu \mathrm{m}$ layer of gold is inserted between the sample and the ablator to filter corona X-rays. The various interfaces were either deposited or glued thanks to formvar membranes to minimize impedance mismatches due to the use of glue. As described above, all these thicknesses were judiciously chosen to reach and maintain a maximum uniform pressure. For the same reasons, we designed a laser pulse shape, as shown in figure 10.a, with a parabolic time profile. Note that, although it is preferable to use monocrystalline diamonds in order to avoid being hindered by its diffraction lines over the entire azimuthal area [39], we carried out these first experiments with polycrystalline diamonds for budgetary reasons. It should also be noted that a $250 \mathrm{~nm}$ coating of aluminum was made on the front face of the diamond ablator to ensure that all the energy from the foot of the laser pulse is deposited on the front face of the target, and not at depth.

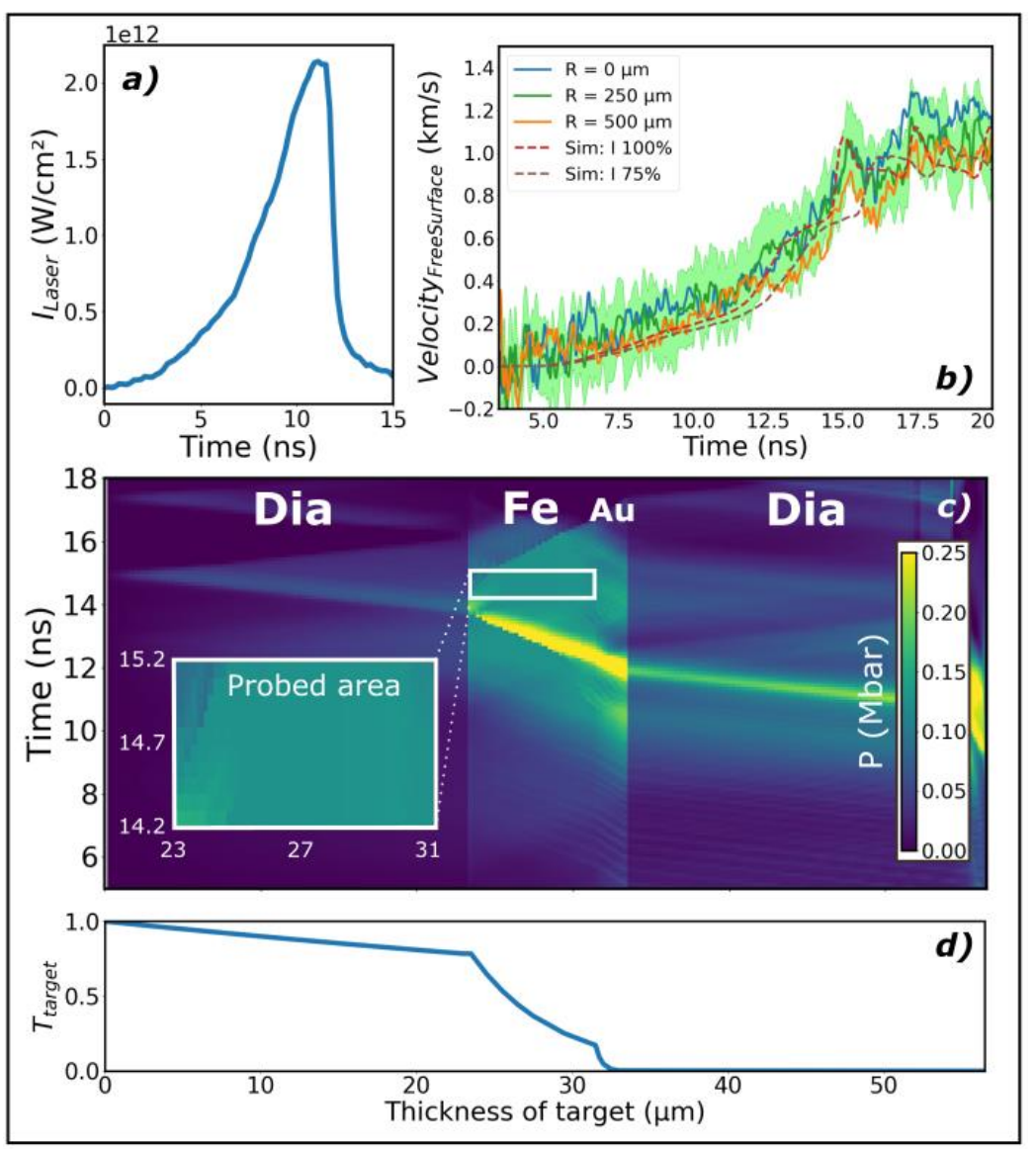


Figure 10: a) Temporal profile of the driver beam used to generate the ramp compression. b) Calculation (dotted line) and measurement (solid lines) of the diamond free-surface velocity as a function of time using VISAR diagnosis, at distance $R$ from the centre of the sample. The green band represents the measurement uncertainties on the velocity in the intermediate probed area. c) Density map obtained by means of a hydrodynamic simulation, which highlights the homogeneous compressed hydrodynamic conditions surveyed in iron. d) Two-way X-ray absorption of the sample at $6.70 \mathrm{keV}$, considering an angle of incidence of $60^{\circ}$.

Using a secondary target, we found that the backside of the diamond, which was not treated with an antireflective coating, was much more reflective than the iron/diamond interface (by a factor of more than 3 ). This may be due to the poor surface condition of the iron layer, which is glued and not coated, and highly diffusing. Consequently, the VISAR mainly record the free surface velocity of the diamond, as shown in Figure 10.b. In addition, using hydrodynamic simulations, we checked that the small amount of signal that could come from the iron/diamond interface did not pose any problem to the interpretation of the VISAR data since the apparent velocity of this interface was very similar to the free surface velocity of the diamond.

For this measurement, we used the same VISAR sensitivities as those used in the previous experiment. Given the low free surface velocities, the large error bars do not allow us to observe significant transverse inhomogeneities in these measurements. Indeed, the expansion velocities measured both in the center (in blue) and at the edge (in orange) of the probed zone are largely included in the error bars of the one measured in the intermediate zone (in green). We can then back-propagate the equations of motion to accurately determine the pressure history within the sample layer, using the characteristics method, as described in [60,61], or using hydrodynamic simulations. We used this second option, having taken care upstream to calibrate the laser deposition in our simulations using specific shots, where the same laser pulse shape was applied on simple Al-coated diamond membranes, as well as on simple iron sheets. The agreement between the measurement and the simulated release of the diamond window shown in the same figure (dotted red line) allows us to give confidence to the simulation. We also note thanks to these simulations that a $25 \%$ reduction of the laser intensity (dotted brown line) has a limited influence on the compression dynamics and that the expected free surface velocities remain very close to those measured at the edge of the focal spot (orange line) despite the large error bars. As presented in the previous section, the simulated density map of this example is shown in Figure 10.c. and allows evaluating the hydrodynamic conditions probed between 14.2 and 15.2 ns, i.e. during the probed time, with the same $\mathrm{He}_{\alpha} \mathrm{X}$-ray source.

Again, the diffracted X-rays collected by IPs came mostly from the first rear side microns of iron due to $\mathrm{X}$-ray absorption in iron, as shown in Figure 10.d. Note also that diamond absorbs a significant part of the X-ray signal $(\approx 20 \%)$ and then diffracts a significant part of the probe. However, with this experimental strategy, the longitudinal hydrodynamic conditions are stable in the sample for more than 2 ns, i.e. for a time greater than that of the probe. In that case, the vast majority of the diffracted signal from iron comes from the white space-time rectangle shown in Figure 10.c, which illustrates the homogeneity of the hydrodynamic conditions surveyed. With an analysis similar to that described in the previous section, the simulation predicts the density and the pressure of the probed compressed iron at $8.59 \pm 0.15 \mathrm{~g} / \mathrm{cm}^{3}$ and $12.6 \pm 0.9 \mathrm{GPa}$. Here again, the temporal and spatial uniformity of the sample density and pressure during X-ray exposure is evaluated based on the variance of the density and stress values in this region.

\subsubsection{Diffraction results}

As presented in Figure 11.a, the corresponding XRD image shows diffraction lines from the diamond window (mainly around standard density), face-centred cubic (fcc) gold from the compressed X-ray shield, as well 
as compressed bcc and hep iron. Calibration of the experimental geometry has been done using diffraction lines of diamond originating from both $\mathrm{Fe}_{\alpha}$ and $\mathrm{Fe} \mathrm{He}_{\beta}$ emissions.

Since the spurious signal from WC collimator does not interfere with diffraction lines from the sample, we have performed azimuthal integration over the entire detector except IP R. Applying the same procedure as described in section 3.1.2, we subtracted a spline baseline to the spectrum and fitted it a sum of Gaussian curves, as shown in Figure 11.b. The corresponding 20-positions and d-spacings are listed in Table 2.

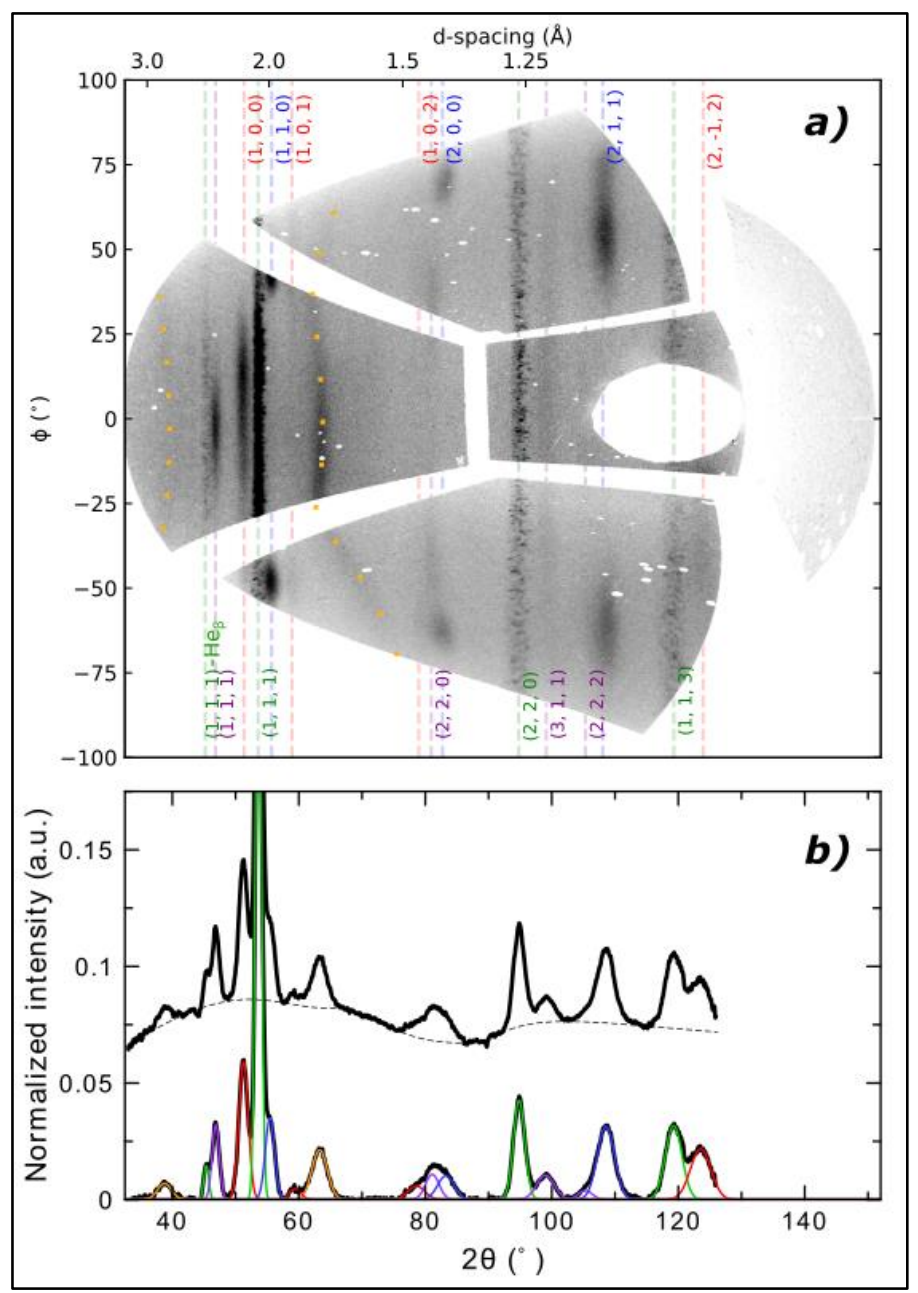

Figure 11: a) Diffraction images of ramp-compressed Fe projected in the $2 \theta-\phi$ plane. Dotted lines show the positions of the (hkl) planes that are visible on the XRD image: green, uncompressed diamond (used to calibrate the experimental geometry); blue, compressed bcc iron; red, compressed hcp iron; purple, compressed fcc gold (X-ray shield). The orange dotted line shows parasitic signal from WC collimator. b) Azimuthal integration and corresponding spline background. The spectrum after background subtraction is shown together with Gaussian curves fitting diamond (green), bcc Fe (blue), hcp Fe (red), fcc gold (purple) and the signal from WC (orange).

From these d-spacings, we deduce that compressed bcc iron has a cell parameter $a_{b c c}=2.805 \pm 0.002 \AA$, which results in a density of $8.400 \pm 0.017 \mathrm{~g} / \mathrm{cm}^{3}$. For hcp-Fe, we obtain $a_{h c p}=2.473 \pm 0.006 \AA$ and c/a $=1.621 \pm$ 0.014 , which corresponds to a density of $8.740 \pm 0.097 \mathrm{~g} / \mathrm{cm}^{3}$. 
Thus, the analysis of the diffraction pattern shows the coexistence of both bcc and hep phases at densities banding the predicted density and at pressure close to the phase transition pressure. Previous studies of rampcompressed iron under similar conditions have shown a characteristic transition time of around $1 \mathrm{~ns}$ [21], therefore a time integrated diffraction over $1 \mathrm{~ns}$ is expected to show lines of both phases. Moreover, the density difference at equilibrium pressure is compatible with density measurements obtained from surface velocity measurements at the onset pressure of the $\alpha-\varepsilon$ transition [20]. A detailed study of the phase transition dynamics would require better temporal resolution, i.e. shorter X-ray burst duration. Such a study is beyond the scope of this article.

\begin{tabular}{|c|cc|}
\hline Plane & $\mathbf{2 \theta}\left(^{\circ}\right)$ & $\mathbf{d}(\boldsymbol{\AA})$ \\
\hline $\mathbf{B C C}-\mathbf{F e}$ & & \\
$(110)$ & $55.43(15)$ & $1.996(5)$ \\
$(200)$ & $83.24(37)$ & $1.397(5)$ \\
$(211)$ & $108.57(15)$ & $1.143(2)$ \\
\hline $\mathbf{H C P}-\mathbf{F e}$ & & \\
$(100)$ & $51.26(15)$ & $2.146(7)$ \\
$(101)$ & $59.42(16)$ & $1.873(4)$ \\
$(102)$ & $78.59(38)$ & $1.465(6)$ \\
$(2-12)$ & $123.54(15)$ & $1.053(1)$ \\
\hline
\end{tabular}

Table 2: $2 \theta$-angles of peak maxima and corresponding $d$-spacings of bcc and hcp Fe (hkl) planes. Values in parenthesis give the error on the last digit.

\section{Summary}

We report the design and performances of a reflection X-ray diffraction platform on dynamically compressed solids at large laser facilities. The sub-nanosecond quasi-monochromatic X-ray source is generated at LULI2000 facility using an intense laser illuminating a thin metallic foil, and penetrates inside the tungsten carbide diffractometer through a collimator whose size can be changed. The diffraction pattern is recorded on a $2 \theta \mathrm{X}$-ray scattering angular coverage between $30^{\circ}$ and $150^{\circ}$, with a precision better than $0.2^{\circ}$. The pressure and temperature history of probed samples is measured in parallel thanks to rear-side visible diagnostics. This geometrical configuration has several advantages: it shields the detectors against the ablation plasma X-ray noise and it allows probing a small portion of the compressed sample. Overall, these improvements facilitate probing spatially and temporarily uniform thermodynamic conditions, making it possible to study the phase changes of materials of interest under different compression dynamics by adapting the thickness of the different materials composing the target, as well as the temporal shape of the laser pulse, as we have shown in the case of iron. Nevertheless, we warn that the reflection geometry requires a finer adjustment of the pump-probe delay, as well as a finer alignment of the setup compare to the transmission geometry. Finally, we note that this experimental setup is currently being studied to be fitted with the Laser MegaJoule facility, associated with X-ray $\mathrm{K}_{\alpha}$ emission generated by PETAL.

\section{Acknowledgments}

The authors would like to thank the LULI laser staff for supporting the experiments, and F. Chermette, X. Vaisseau, V. Svitlyk, S. Baton, Ch. Rousseaux, C. Reverdin, and B. Vauzour for their precious technical help and 
discussions. J.-A.H was supported by the Agence Nationale de la Recherche (ANR) under project number ANR16-CE21-0008 (POMPEI) and by the Research Council of Norway through its Centres of Excellence funding scheme, project number 223272.

\section{Data availability}

The data that supports the findings of this study are available within the article.

\section{References}

[1] M. H. Rice, R. G. McQueen and J. M. Walsh, Compression of Solids by Strong Shock Waves, Solid State Physics 6, 1-63 (1958).

[2] L. Davison and R. A. Graham, Shock compression of solids, Physics Reports 55, 4, 255-379 (1979).

[3] B. D. Cullity and S. R. Stock, Elements of X-ray diffraction, Addison.Wesley Publishills Ca., Inc., Reading, Mass., (1956).

[4] B. E. Warren, X-ray diffraction, Dover Publications, (1969).

[5] H. P. Klug and L. E. Alexander, X-ray Diffraction Procedures for Polycrystalline and Amorphous Materials, $2^{\text {nd }}$ Edition, pp. 992. ISBN 0-471-49369-4. Wiley-VCH, (1974).

[6] J. Hu, K. Ichiyanagi, T. Doki, A. Goto, T. Eda, K. Norimatsu, S. Harada, D. Horiuchi, Y. Kabasawa, S. Hayashi, S. Uozumi, N. Kawai, S. Nozawa, T. Sato, S. Adachi, and K. G. Nakamura, Complex structural dynamics of bismuth under laser-driven compression, Appl. Phys. Lett 103, 161904 (2013).

[7] A. E. Gleason, C. A. Bolme, H. J. Lee, B. Nagler, E. Galtier, D. Milathianaki, J. Hawreliak, R. G. Kraus, J. H. Eggert, D. E. Fratanduono, G. W. Collins, R. Sandberg, W. Yang, and W. L. Mao, Ultrafast visualization of crystallization and grain growth in shock-compressed $\mathrm{SiO}_{2}$, Nat. Commun. 6, 819 (2015).

[8] M. G. Gorman, R. Briggs, E. E. McBride, A. Higginbotham, B. Arnold, J. H. Eggert, D. E. Fratanduono, E. Galtier, A. E. Lazicki, H. J. Lee, H. P. Liermann, B. Nagler, A. Rothkirch, R. F. Smith, D. C. Swift, G. W. Collins, J. S. Wark, and M. I. McMahon, Direct Observation of Melting in Shock-Compressed Bismuth With Femtosecond X-ray Diffraction, Phys. Rev. Lett. 115, 095701 (2015).

[9] D. Kraus, A. Ravasio, M. Gauthier, D. O. Gericke, J. Vorberger, S. Frydrych, J. Helfrich, L. B. Fletcher, G. Schaumann, B. Nagler, B. Barbrel, B. Bachmann, E. J. Gamboa, S. Göde, E. Granados, G. Gregori, H. J. Lee, P. Neumayer, W. Schumaker, T. Döppner, R. W. Falcone, S. H. Glenzer, and M. Roth, Nanosecond formation of diamond and lonsdaleite by shock compression of graphite, Nat. Commun. 7, 10970 (2016)

[10] R. Briggs, M. G. Gorman, A. L. Coleman, R. S. McWilliams, E. E. McBride, D. McGonegle, J. S. Wark, L. Peacock, S. Rothman, S. G. Macleod, C. A. Bolme, A. E. Gleason, G. W. Collins, J. H. Eggert, D. E. Fratanduono, R. F. Smith, E. Galtier, E. Granados, H. J. Lee, B. Nagler, I. Nam, Z. Xing, and M. I. McMahon, Ultrafast X-Ray Diffraction Studies of the Phase Transitions and Equation of State of Scandium Shock Compressed to 82 GPa, Phys. Rev. Lett. 118, 025501 (2017).

[11] D. Kraus, J. Vorberger, A. Pak, N. J. Hartley, L. B. Fletcher, S. Frydrych, E. Galtier, E. J. Gamboa, D. O. Gericke, S. H. Glenzer, E. Granados, M. J. MacDonald, A. J. MacKinnon, E. E. McBride, I. Nam, P. Neumayer, M. Roth, A. M. Saunders, A. K. Schuster, P. Sun, T. van Driel, T. Döppner, and R. W. Falcone, Formation of diamonds in laser-compressed hydrocarbons at planetary interior conditions, Nature Astro. 1, 606 (2017). 
[12] A. E. Gleason, C. A. Bolme, E. Galtier, H. J. Lee, E. Granados, D. H. Dolan, C. T. Seagle, T. Ao, S. Ali, A. Lazicki, D. Swift, P. Celliers, and W. L. Mao, Compression Freezing Kinetics of Water to Ice VII, Phys. Rev. Lett. 119, 025701 (2017).

[13] A. E. Gleason, C. A. Bolme, H. J. Lee, B. Nagler, E. Galtier, R. G. Kraus, R. Sandberg, W. Yang, F. Langenhorst, and W. L. Mao, Time-resolved diffraction of shock-released SiO2 and diaplectic glass formation, Nat. Commun. 8, 1481 (2017).

[14] R. Briggs, R. Torchio, A. Sollier, F. Occelli, L. Videau, N. Kretzschmara and M. Wulff, Observation of the shock-induced $\beta$-Sn to b.c.t.-Sn transition using time-resolved X-ray diffraction, Journal of Synchrotron Radiation 26, 96-101 (2019)

[15] C. M. Pépin, A. Sollier, A. Marizy, F. Occelli, M. Sander, R. Torchio, and P. Loubeyre, Kinetics and structural changes in dynamically compressed bismuth, Phys. Rev. B 100, 060101(R) (2019).

[16] A. Benuzzi-Mounaix, S. Mazevet, A. Ravasio, T. Vinci, A. Denoeud, M. Koenig, N. Amadou, E. Brambrink, F. Festa, A. Levy, M. Harmand, S. Brygoo, G. Huser, V. Recoules, J. Bouchet, G. Morard, F. Guyot, T. de Resseguier, K. Myanishi, N. Ozaki, F. Dorchies, J. Gaudin, P.-M. Leguay, O. Peyrusse, O. Henry, D. Raffestin, S. Le Pape, R. Smith, and R. Musella, Progress in Warm Dense Matter study with applications to planetology, Physica Scripta 161, 0144060 (2014).

[17] K. Falk, Experimental methods for warm dense matter research, High Power Laser Science and Engineering 6, e59 (2018).

[18] T. Duffy and R. Smith, Ultra-high pressure dynamic compression of geological materials, Frontiers in Earth Science 7, 23 (2019).

[19] R. F. Smith, J. H. Eggert, M. D. Saculla, A. F. Jankowski, M. Bastea, D. G. Hicks, and G. W. Collins, Ultrafast Dynamic Compression Technique to Study the Kinetics of Phase Transformations in Bismuth, Phys. Rev. Lett 101, 065701 (2008).

[20] R. F. Smith, J. H. Eggert, D. C. Swift, J. Wang, T. S. Duffy, D. G. Braun, R. E. Rudd, D. B. Reisman, J.-P. Davis, M. D. Knudson, and G. W. Collins, Time-dependence of the alpha to epsilon phase transformation in iron, Journal of Applied Physics 114, 223507 (2013).

[21] N. Amadou, T. de Resseguier, E. Brambrink, T. Vinci, A. Benuzzi-Mounaix, G. Huser, G. Morard, F. Guyot, K. Miyanishi, N. Ozaki, R. Kodama, and M. Koenig, Kinetics of the iron a-e phase transition at high-strain rates, Phys. Rev. B. 93, 214108 (2016).

[22] T. Feurer, A. Morak, I. Uschmann, Ch. Ziener, H. Schwoerer, Ch. Reich, P. Gibbon, E. Förster, R. Sauerbrey, K. Ortner, and C. R. Becker, Femtosecond silicon K-alpha pulses from laser-produced plasmas, Phys. Rev. E 65, $016412(2001)$.

[23] J. Workman and G. A. Kyrala, Scaling of $x$-ray K-shell sources from laser-solid interactions, Proc. SPIE 4504, 168 (2001).

[24] F. Girard, Review of laser produced multi-keV X-ray sources from metallic foils, cylinders with liner, and low density aerogels, Physics of Plasmas 23, 040501 (2016).

[25] F. Coppari , R. F. Smith, D. B. Thorn, J. R. Rygg, D. A. Liedahl, R. G. Kraus, A. Lazicki, M. Millot, and J. H. Eggert, Optimized $x$-ray sources for x-ray diffraction measurements at the Omega Laser Facility, Review of Scientific Instruments 90, 125113 (2019). 
[26] L. M. Barker and R. E. Hollenbach, Laser interferometer for measuring high velocities of any reflecting surface, J. Appl. Phys. 43, 4669 (1972).

[27] J. E. Miller, T. R. Boehly, A. Melchior, and D. D. Meyerhofer, Streaked optical pyrometer system for laserdriven shock-wave experiments on OMEGA, Rev. Sci. Instrum. 78, 034903 (2007).

[28] A. L. Meadowcroft, C. D. Bentley, and E. N. Stott, Evaluation of the sensitivity and fading characteristics of an image plate system for x-ray diagnostics, Rev. Sci. Instrum. 79, 113102 (2008).

[29] B. R. Maddox, H. S. Park, B. A. Remington, N. Izumi, S. Chen, C. Chen, G. Kimminau, Z. Ali, M. J. Haugh, and Q. Ma, High-energy x-ray backlighter spectrum measurements using calibrated image plates, Rev. Sci. Instrum. 82, 023111 (2011).

[30] T. Bonnet, M. Comet, D. Denis-Petit, F. Gobet, F. Hannachi, M. Tarisien, M. Versteegen, and M. M. Aléonard, Response functions of imaging plates to photons, electrons and ${ }^{4} H e$ particles, Rev. Sci. Instrum. 84, 103510 (2013).

[31] J. Wark, R. Whitlock, A. Hauer, J. Swain, and P. Solone, Shock launching in silicon studied with use of pulsed X-ray-diffraction, Phys. Rev. B 35, 9391-9394 (1987).

[32] J. Wark, R. Whitlock, A. Hauer, J. Swain, and P. Solone, Subnanosecond x-ray diffraction from laser-shocked crystals, Phys. Rev. B 40, 5705 (1989).

[33] D. H. Kalantar, J. F. Belak, G. W. Collins, J. D. Colvin, H. M. Davies, J. H. Eggert, T. C. Germann, J. Hawreliak, B. L. Holian, K. Kadau, P. S. Lomdahl, H. E. Lorenzana, M. A. Meyers, K. Rosolankova, M. S. Schneider, J. Sheppard, J. S. Stölken, and J. S. Wark, Direct observation of the alpha-epsilon transition in shock compressed iron via nanosecond X-ray diffraction, Phys. Rev. Lett. 95, 075502 (2005).

[34] J. Hawreliak, J. D. Colvin, J. H. Eggert, D. H. Kalantar, H. E. Lorenzana, J. S. Stölken, H. M. Davies, T. C. Germann, B. L. Holian, K. Kadau, P. S. Lomdahl, A. Higginbotham, K. Rosolankova, J. Sheppard, and J. S. Wark, Analysis of the X-ray diffraction signal for the alpha-epsilon transition in shock-compressed iron: Simulation and experiment, Phys. Rev. B 74, 184107 (2006).

[35] J. Hawreliak, B. El-Dasher, H. Lorenzana, G. Kimminau, A. Higginbotham, B. Nagler, S. Vinko, W. Murphy, T. Whitcher, J. Wark, S. Rothman, and N. Park, In situ X-ray diffraction measurements of the cla ratio in the highpressure epsilon phase of shock-compressed polycrystalline iron, Phys. Rev. B 83, 144114 (2011).

[36] A. Denoeud, N. Ozaki, A. Benuzzi-Mounaix, H. Uranishi, Y. Kondo, R. Kodama, E. Brambrink, A. Ravasio, M. Bocoum, J.-M. Boudenne, M. Harmand, F. Guyot, S. Mazevet, D. Riley, M. Makita, T. Sano, Y. Sakawa, Y. Inubushi, G. Gregori, M. Koenig, and G. Morard, Dynamic X-ray diffraction observation of shocked solid iron up to 170 GPa, Proc Natl Acad Sci USA 113, 28, 7745-7749 (2016).

[37] A. Higginbotham, S. Patel, J. A. Hawreliak, O. Ciricosta, G. W. Collins, F. Coppari, J. H. Eggert, M. J. Suggit, H. Tang, and J. S. Wark, Single photon energy dispersive x-ray diffraction, Rev. Sci. Instrum 85, 033906 (2014).

[38] R. Briggs, M. J. Suggit, M. G. Gorman, A. Coleman, R. Heathcote, A. Higginbotham, S. Patel, J. S. Wark, and M. I. McMahon, Phase transitions in shock compressed bismuth identified using single photon energy dispersive X-ray diffraction (SPEDX), J. Phys.: Conf. Ser. 950042038 (2017).

[39] J. R. Rygg, J. H. Eggert, A. E. Lazicki, F. Coppari, J. A. Hawreliak, D. G. Hicks, R. F. Smith, C. M. Sorce, T. M. Uphaus, B. Yaakobi, and G. W. Collins, Powder diffraction from solids in the terapascal regime, Rev. Sci. Instrum 83, 113904 (2012).

[40] J. R. Rygg, R. F. Smith, A. E. Lazicki, D. G. Braun, D. E. Fratanduono , R. G. Kraus, J. M. McNaney, D. C. Swift, C. E. Wehrenberg, F. Coppari , M. F. Ahmed, M. A. Barrios , K. J. M. Blobaum, G. W. Collins, A. L. Cook, P. Di Nicola, E. G. Dzenitis, S. Gonzales, B. F. Heidl, M. Hohenberger, A. House, N. Izumi, D. H. Kalantar, S. F. 
Khan, T. R. Kohut, C. Kumar, N. D. Masters, D. N. Polsin, S. P. Regan, C. A. Smith, R. M. Vignes, M. A. Wall, J. Ward, J. S. Wark, T. L. Zobrist, A. Arsenlis, and J. H. Eggert, X-ray diffraction at the National Ignition Facility, Rev. Sci. Instrum 91, 043902 (2020).

[41] F. Coppari, R. F. Smith, J. H. Eggert, J. Wang, J. R. Rygg, A. Lazicki, J. A. Hawreliak, G. W. Collins, and T. S. Duffy, X-ray diffraction study of a new phase of $\mathrm{MgO}$ at exo-planet interior pressures, Nat. Geosci. 6, 926-929 (2013).

[42] A. Lazicki, J. R. Rygg, F. Coppari, R. Smith, D. Fratanduono, R. G. Kraus, G. W. Collins, R. Briggs, D. G. Braun, D. C. Swift, and J. H. Eggert, X-Ray Diffraction of Solid Tin to 1.2 TPa, Phys. Rev. Lett. 115, 075502 (2015).

[43] J. Wang, F. Coppari, R. F. Smith, J. H. Eggert, A. E. Lazicki, D. E. Fratanduono, J. R. Rygg, T. R. Boehly, G. W. Collins, and T. S. Duffy, X-ray diffraction of molybdenum under ramp compression to 1 TPa, Phys. Rev. B 94, $104102(2016)$.

[44] J. K. Wicks, R. F. Smith, D. E. Fratanduono, F. Coppari, R. G. Kraus, M. G. Newman, J. R. Rygg, J. H. Eggert, and T. S. Duffy, Crystal structure and equation of state of Fe-Si alloys at super-Earth core conditions, Sci. Adv. 4, eaao5864 (2018).

[45] D. N. Polsin, High-pressure phase transformations of ramp-compressed aluminum and sodium., $\mathrm{PhD}$ thesis http://hdl.handle.net/1802/34287 (2018).

[46] D. N. Polsin, D. E. Fratanduono, J. R. Rygg, A. Lazicki, R. F. Smith, J. H. Eggert, M. C. Gregor, B. J. Henderson, X. Gong, J. A. Delettrez, R. G. Kraus, P. M. Celliers, F. Coppari, D. C. Swift, C. A. McCoy, C. T. Seagle, J.-P. Davis, S. J. Burns, G. W. Collins, and T. R. Boehly, X-ray diffraction of ramp-compressed aluminum to 475 GPa, Phys. Plasmas 25, 082709 (2018).

[47] M. Millot, F. Coppari, J. R. Rygg, A. C. Barrios, S. Hamel, D. C. Swift, and J. H. Eggert, Nanosecond X-ray diffraction of shock-compressed superionic water ice, Nature 569, 251-255 (2019).

[48] J. M. Foster, A. J. Comley, G. S. Case, P. Avraam, S. D. Rothman, A. Higginbotham, E. K. R. Floyd, E. T. Gumbrell, J. J. D. Luis, D. McGonegle, N. T. Park, L. J. Peacock, C. P. Poulter, M. J. Suggit, and J. S. Wark, Xray diffraction measurements of plasticity in shock-compressed vanadium in the region of 10-70 GPa, NatureJ. Appl. Phys. 122, 025117 (2017).

[49] N. Blanchot, G. Behar, T. Berthier, B. Busserole, C. Chappuis, C. Damiens-Dupont, P. Garcia, F. Granet, C. Grosset-Grange, J.-P. Goossens, L. Hilsz, F. Laborde, T. Lacombe, F. Laniesse, E. Lavastre, J. Luce, F. Macias, E. Mazataud, J. L. Miquel, J. Néauport, S. Noailles, P. Patelli, E. Perrot-Minot, C. Present, D. Raffestin, B. Remy, C. Rouyer and D. Valla, Overview of PETAL, the multi-Petawatt project in the LMJ facility, EPJ Web of Conferences 59, 07001 (2013)

[50] S. Glenzer and R. Redmer, X-ray Thomson scattering in high energy density plasmas, Reviews of modern physics 81, 1625-1663 (2009).

[51] J. Kieffer and D. Karkoulis, PyFAI, a versatile library for azimuthal regrouping, Journal of Physics: Conference Series 425, 202012 (2013).

[52] G. Ashiotis, A. Deschildre, Z. Nawaz, J. P. Wright, D. Karkoulis, F. E. Picca, and J. Kieffer, The fast azimuthal integration Python library: pyFAI, Journal of applied crystallography 48(2), 510-519 (2015).

[53] J. Kieffer, V. Valls, N. Blanc, and C. Hennig, New tools for calibrating diffraction setups, Journal of Synchrotron Radiation 27(2) (2020). 
[54] W. G. Wyckoff, Crystal Structures, Interscience Publisher (New York), (1963).

[55] A. Benuzzi-Mounaix, M. Koenig, G. Huser, B. Faral, D. Batani, E. Henry, M. Tomasini, B. Marchet, T. A. Hall, M. Boustie, Th. De Rességuier, M. Hallouin, F. Guyot, D. Andrault, and Th. Charpin, Absolute equation of state measurements of iron using laser driven shocks, Phys. of Plasmas 9, 2466 (2002).

[56] J.F. Colombier, P. Combis, F. Bonneau, R. Le Harzic, and E. Audouard, Hydrodynamic simulations of metal ablation by femtosecond laser irradiation, Phys. Rev. B 71, 165406 (2005).

[57] G.I. Kerley, Multiphase equation of state for iron, Tech. Rep. SAND93-0027, Sandia National Laboratories, Albuquerque, NM (1993).

[58] M. Harmand, A. Ravasio, S. Mazevet, J. Bouchet, A. Denoeud, F. Dorchies, Y. Feng, C. Fourment, E. Galtier, J. Gaudin, F. Guyot, R. Kodama, M. Koenig, H. J. Lee, K. Miyanishi, G. Morard, R. Musella, B. Nagler, M. Nakatsutsumi, N. Ozaki, V. Recoules, S. Toleikis, T. Vinci, U. Zastrau, D. Zhu, and A. Benuzzi-Mounaix, X-ray absorption spectroscopy of iron at multimegabar pressures in laser shock experiments, Physical Review B 92, 024108 (2015).

[59] R. Torchio, F. Occelli, O. Mathon, A. Sollier, E. Lescoute, L. Videau, T. Vinci, A. Benuzzi-Mounaix, J. Headspith, W. Helsby, S. Bland, D. Eakins, D. Chapman, S. Pascarelli, and P. Loubeyre, Probing local and electronic structure in Warm Dense Matter: single pulse synchrotron x-ray absorption spectroscopy on shocked Fe, Scientific Reports 6, 26402 (2015)

[60] J. R. Maw, A Characteristics Code for Analysis of Isentropic Compression Experiments, AIP Conf. Proc. 706, 1217-1220 (2004).

[61] S. D. Rothman and J. Maw, Characteristics analysis of Isentropic Compression Experiments (ICE), J. Phys. IV 134, 745-750 (2006). 

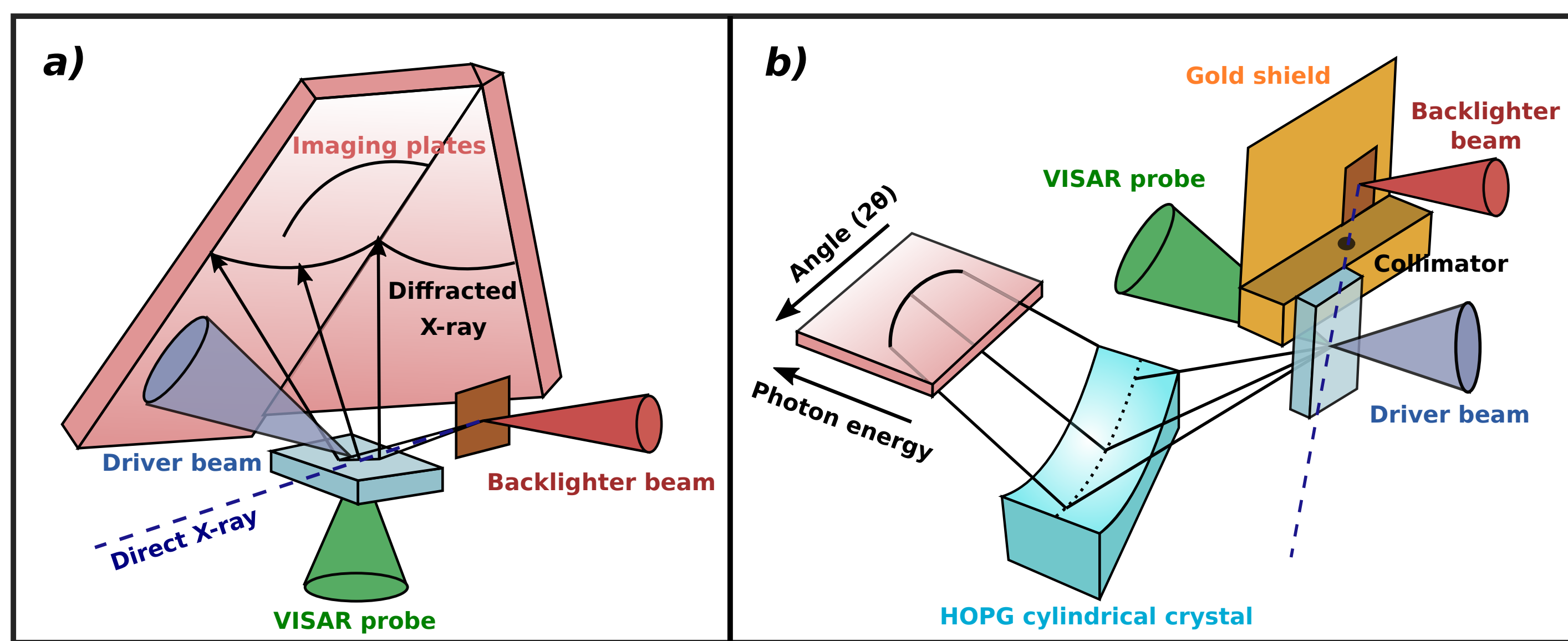

C) Backlighter beam

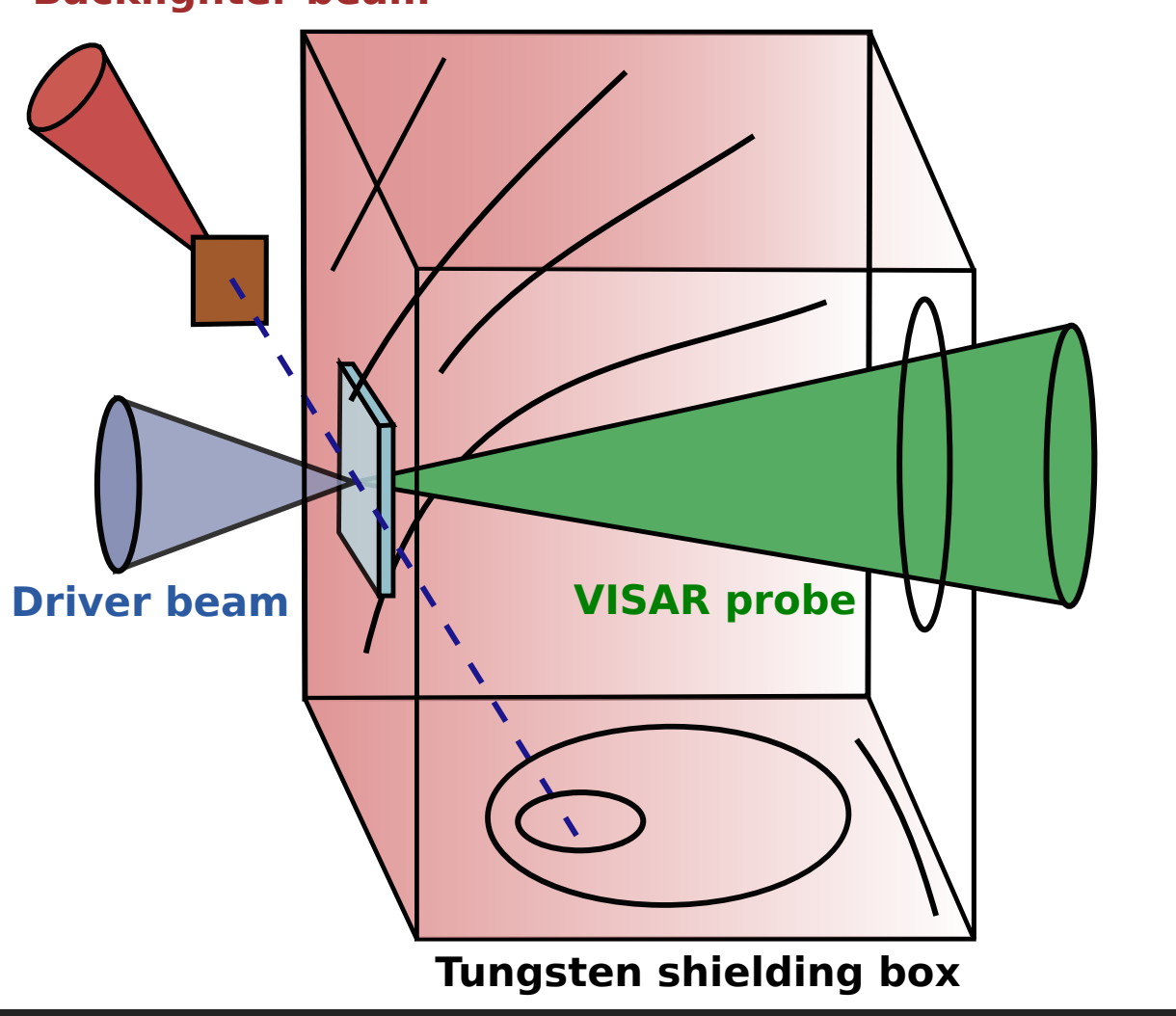




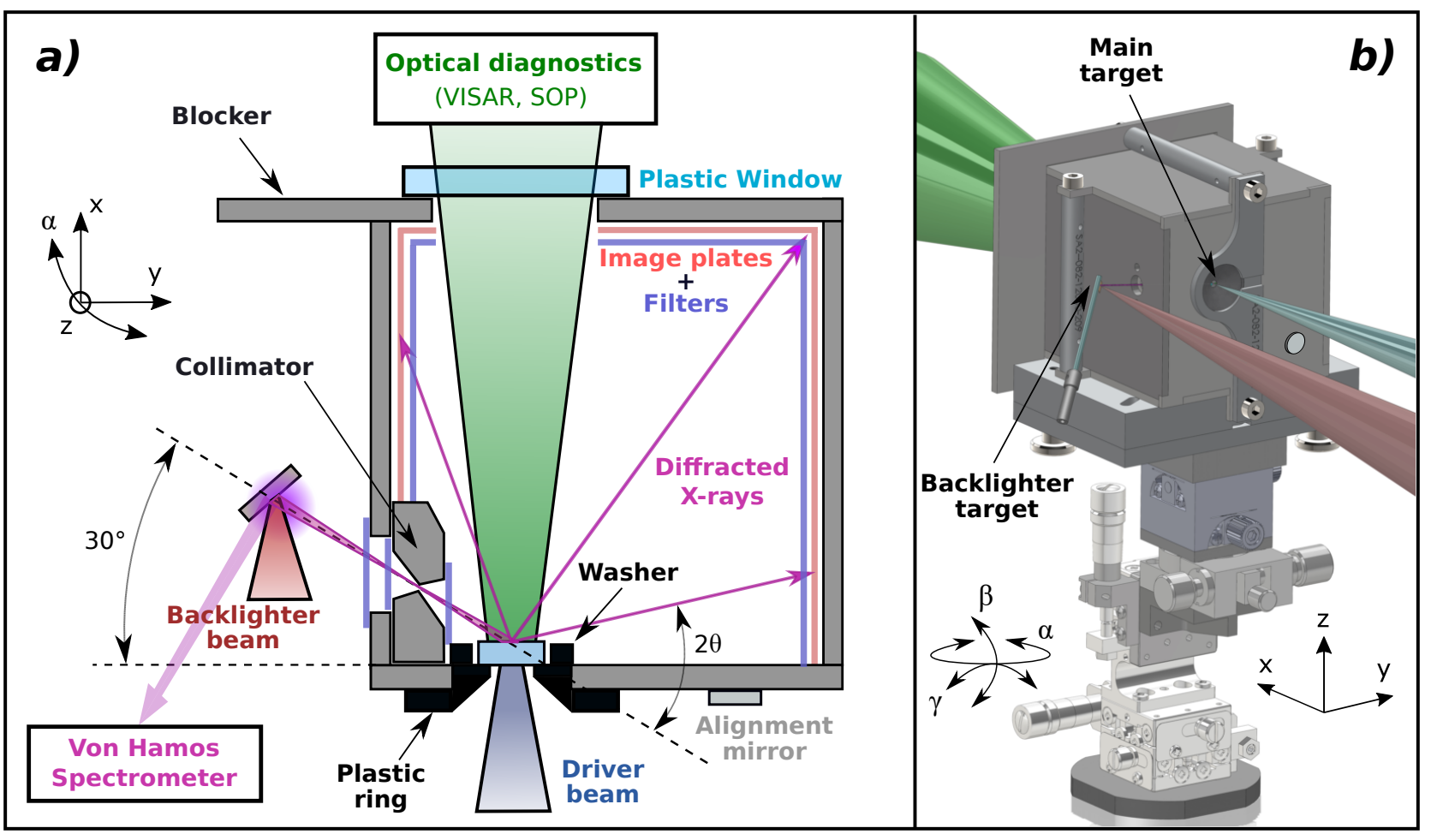



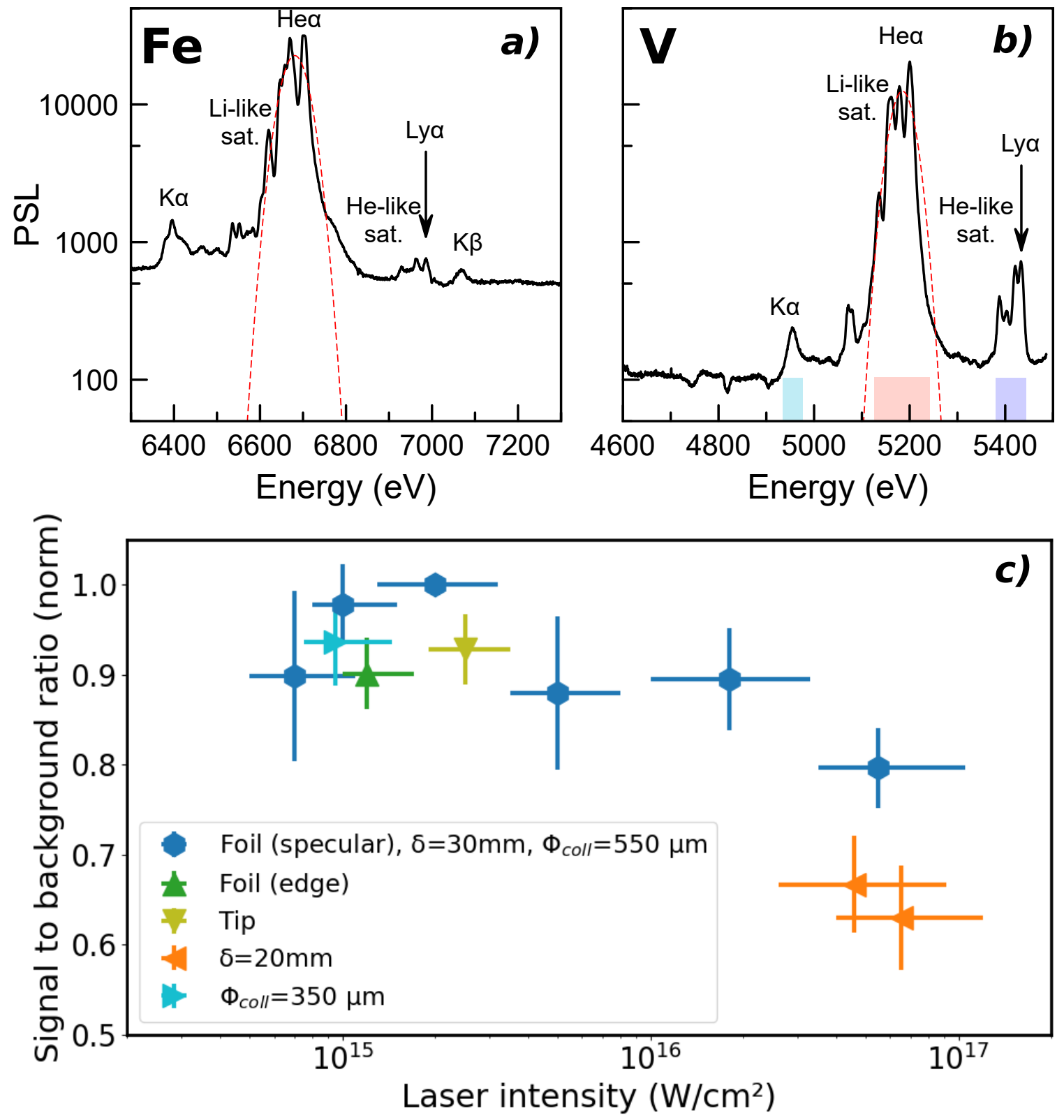


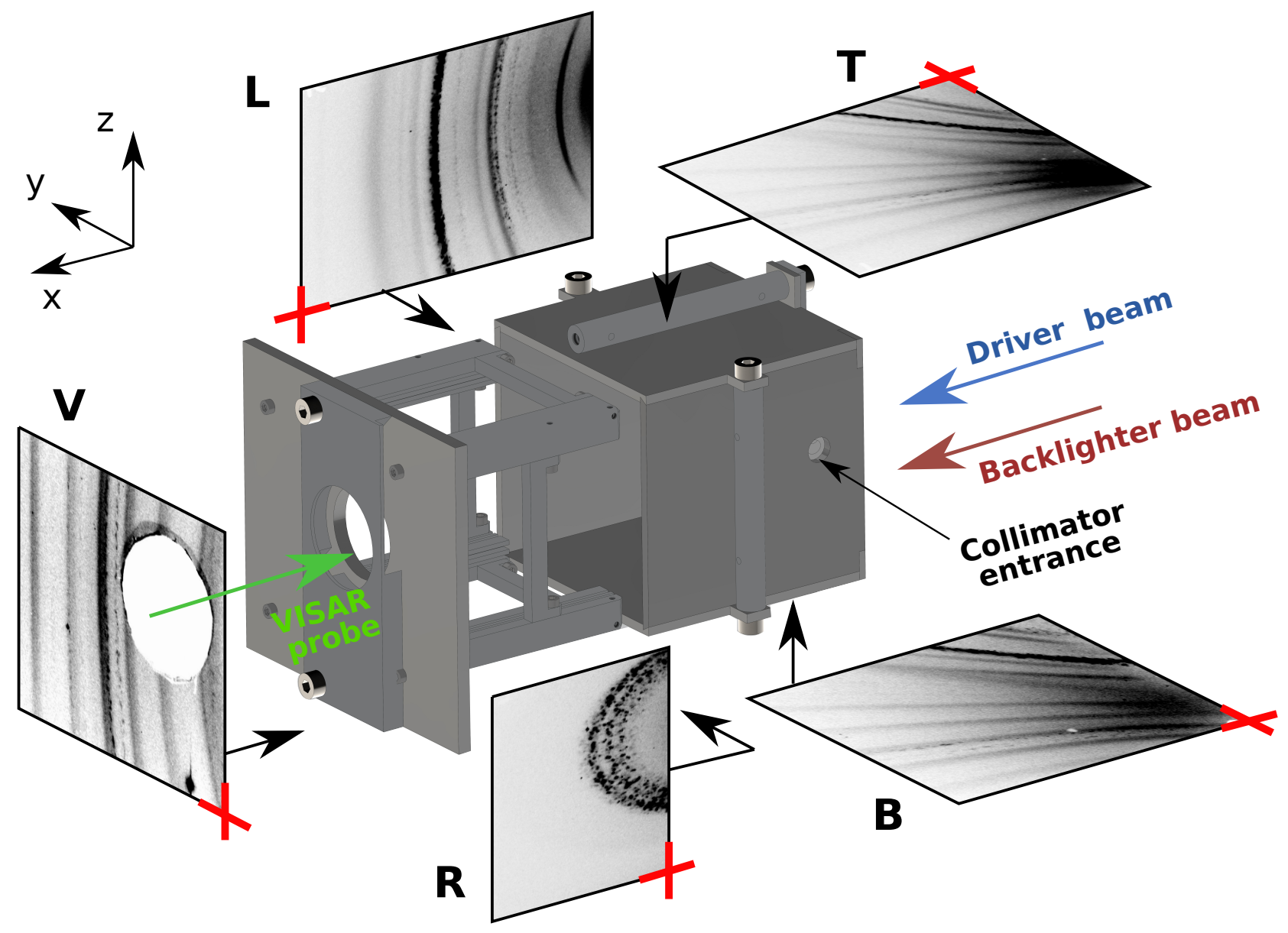



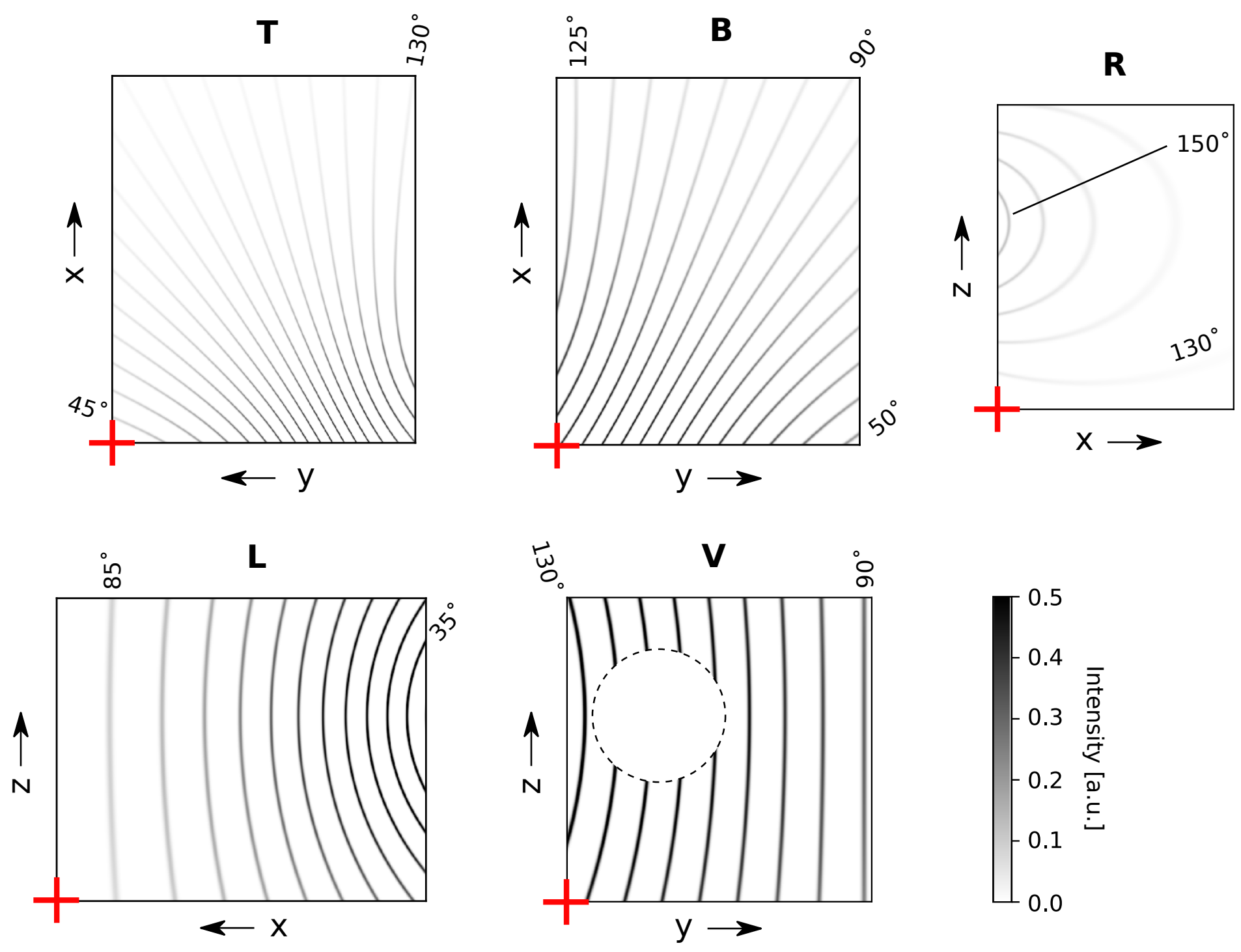

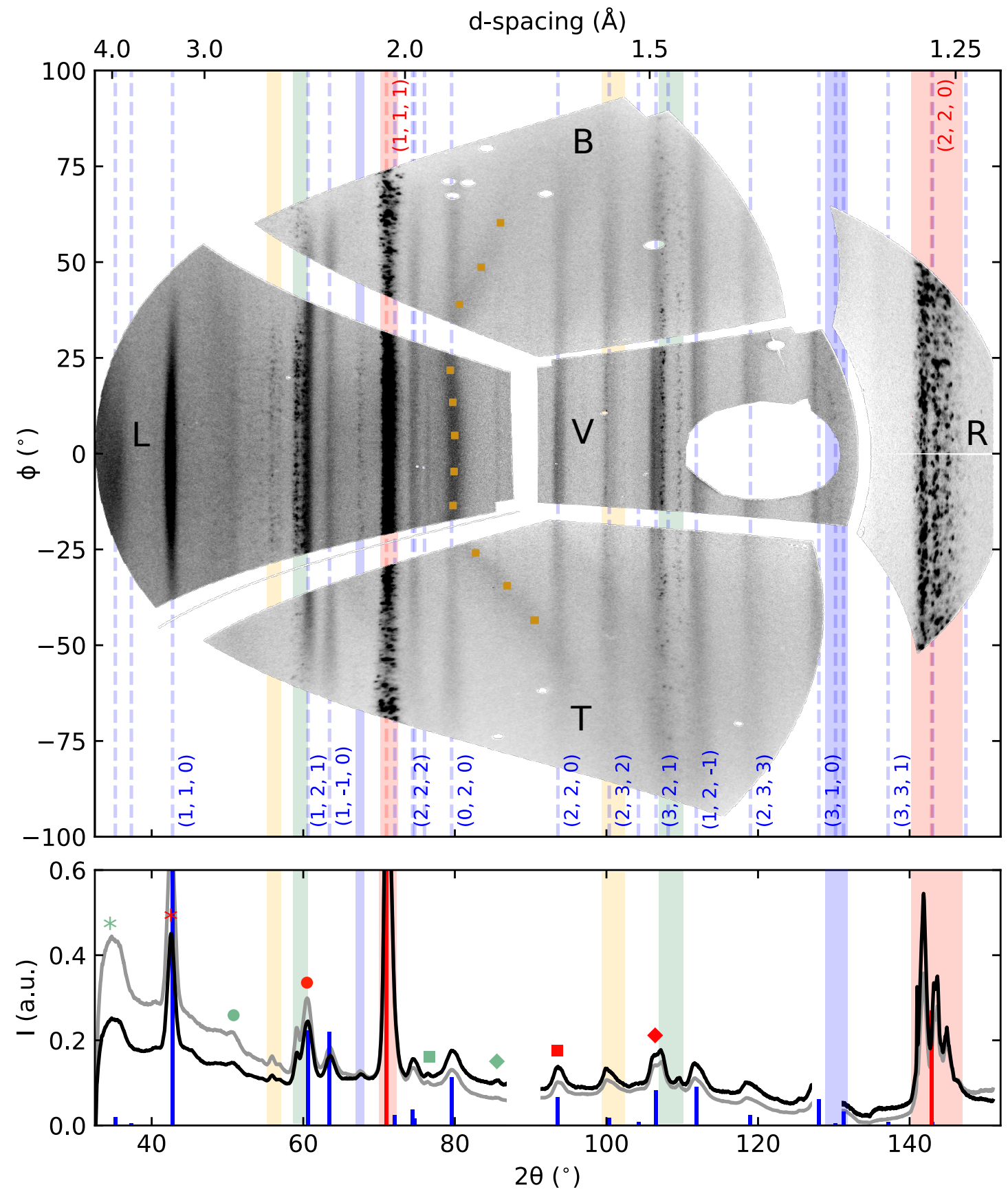

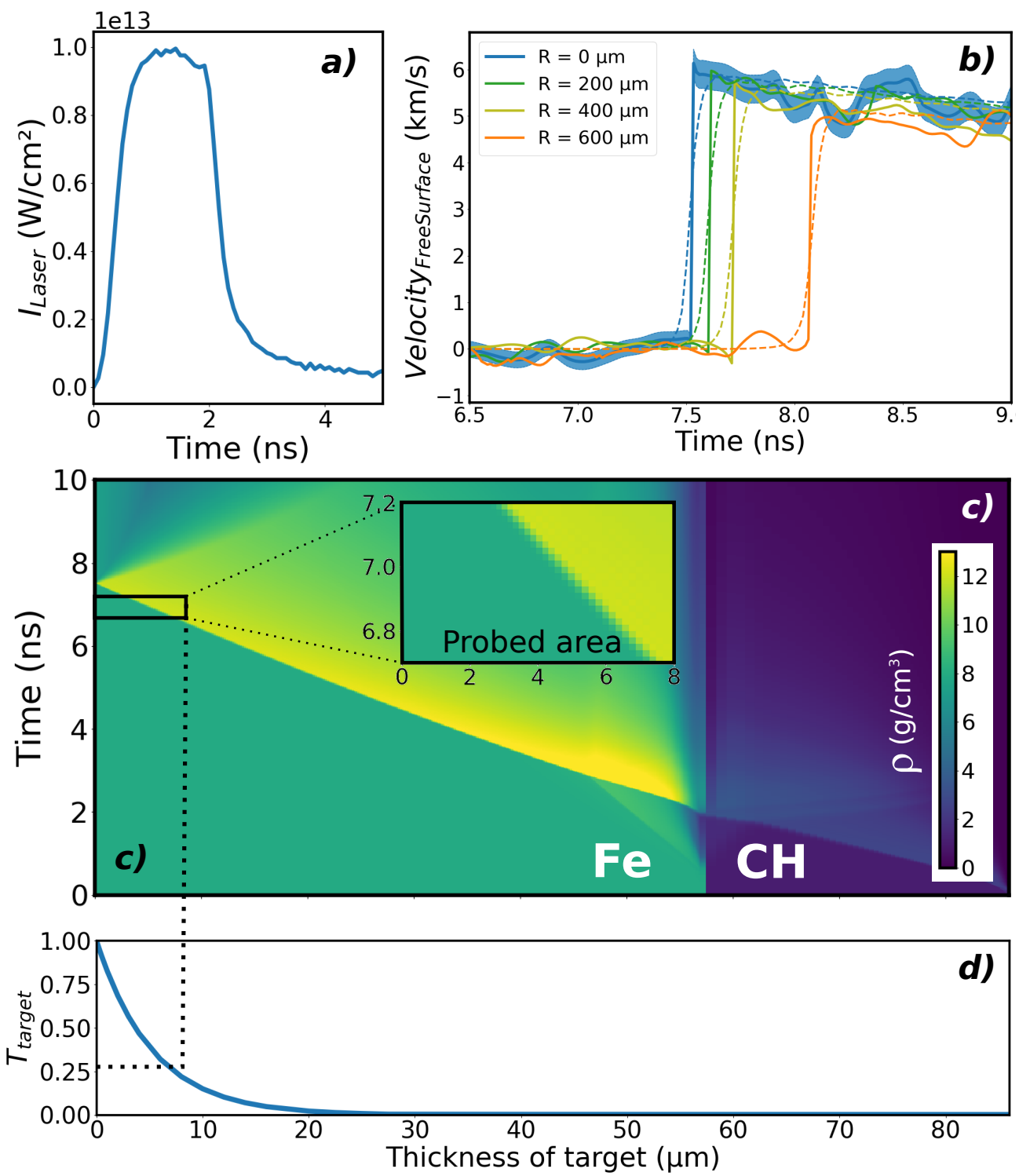

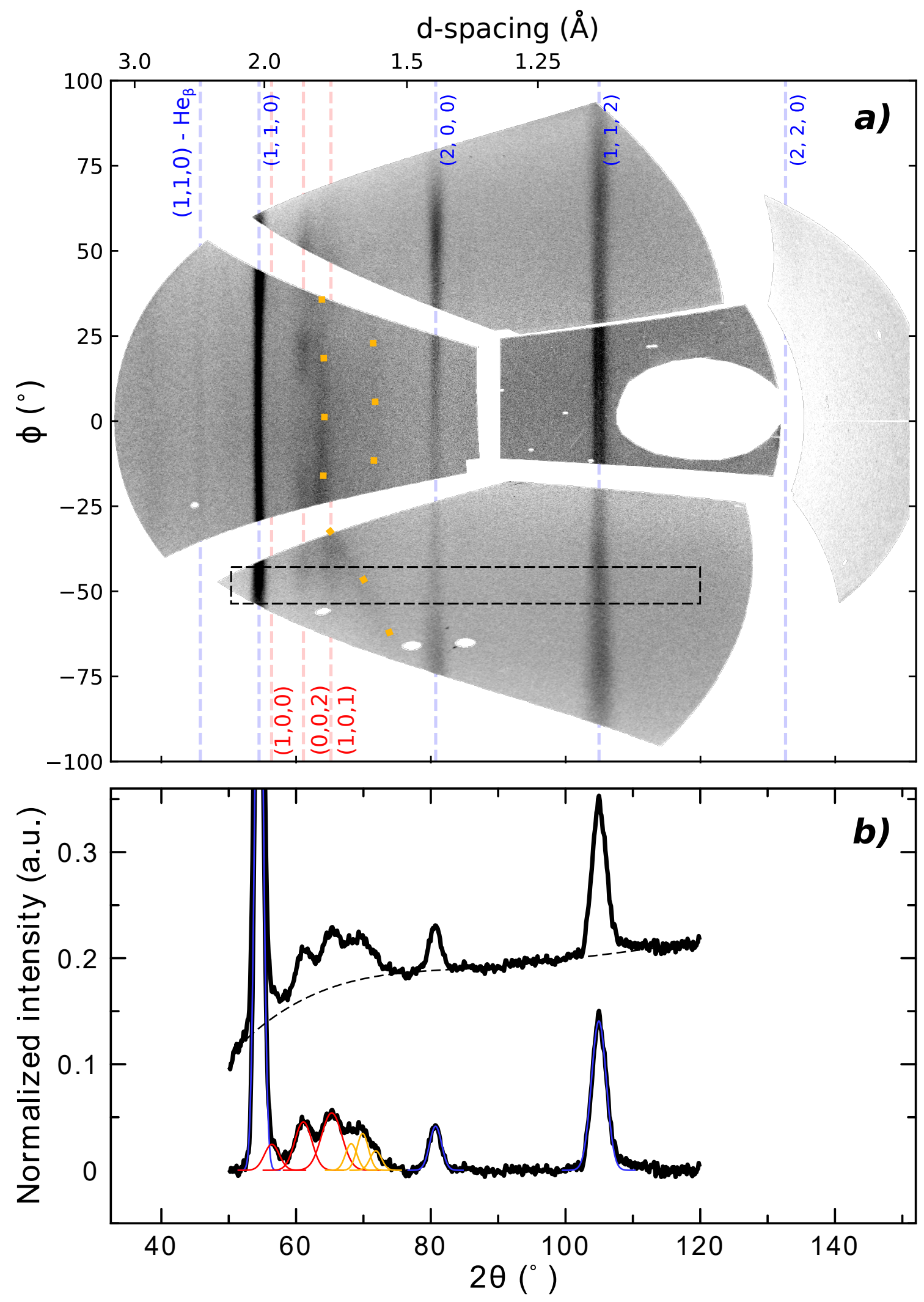

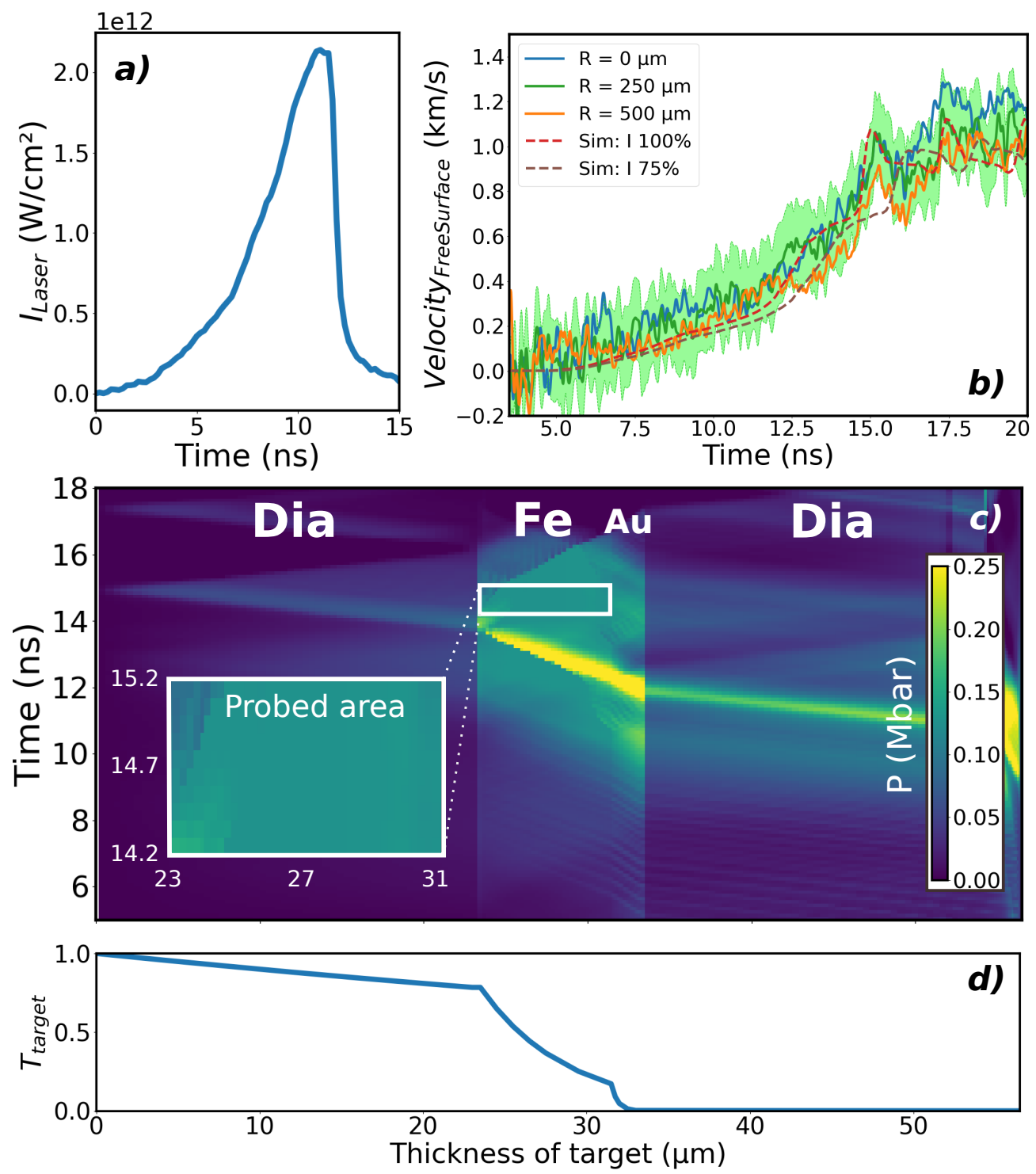

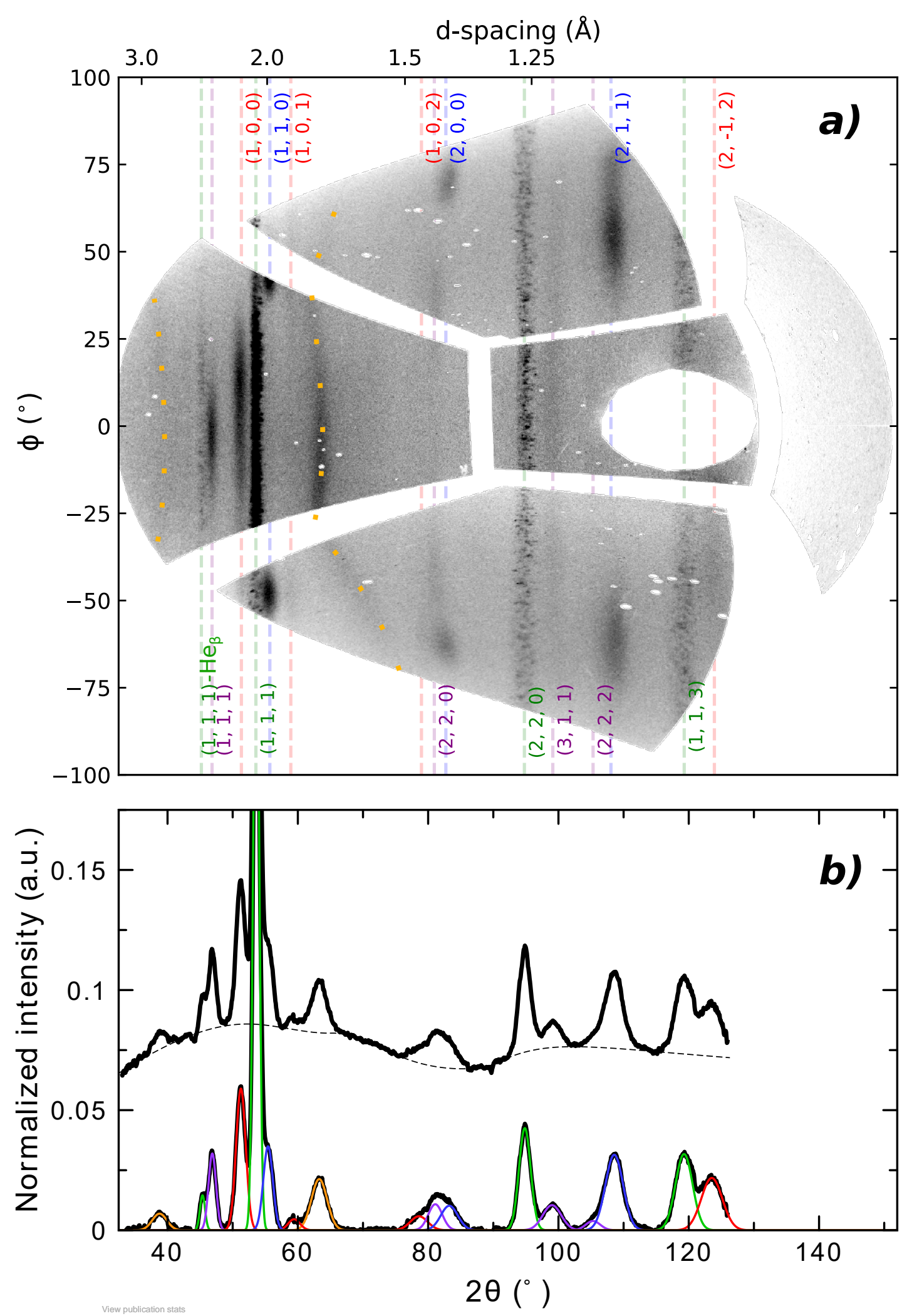